Journal of Mathematics and Statistics 8 (2): 198-210, 2012

ISSN 1549-3644

(C) 2012 Science Publications

\title{
On the Theory of Intuitionistic Fuzzy N-Inner Product Spaces with Applications
}

\author{
Renu Chugh and Seema Mehra \\ Department of Mathematics, \\ Maharshi Dayanand University, Rohtak-124001, India
}

\begin{abstract}
Problem statement: The purpose of this study was to introduce the notion of Intuitionistic fuzzy n-inner product space and to prove a fixed point theorem in complete intuitionistic fuzzy ninner product space. Conclusion/Recommendations: Further this result is applied to obtain the existence and uniqueness of solution for linear volterra integral equation.
\end{abstract}

Key words: Volterra integral equation, intuitionistic fuzzy ninner, complete intuitionistic fuzzy, fixed point theorem, linear space respectively, intuitionistic fuzzy

\section{INTRODUCTION}

An interesting theory of 2-inner product space and n-inner product space has been effectively constructed by (Diminnie et al., 1977). It was further investigated and developed by (Misiak 1989a). Recent results about n-inner product space can be viewed in (Misiak, 1989b; Cho et al., 2002; Cho, 2001). Gunawan and Mashadi 1986) and Malceski (1997), there is a study about the origin and development of n-normed linear space. Fuzzy set theory is useful tool to describe situation in which the data are imprecise or vague. Fuzzy set theory was formalized by Professor (Zadeh, 1965) at the University of California in 1965. After that a lot of studies have been done regarding fuzzy set. The concept of fuzzy set theory may have very important applications in quantum particle physics, medical diagnosis, traffic control system and information retrieval. Different authors introduced the definitions of fuzzy inner product space in (El-Abyad and ElHamauly, 1991; Cheng and Mordeson, 1994) and fuzzy normed linear space in (Narayanan and Vijayabalaji, 2005; Felbin, 1992; 1993; 1999; Krishna and Sarma, 1994; Bag and Samanta, 2003; Gahler, 1964; Katsaras, 1984; Rhie et al., 1997).

Atanassov (1986) introduced and studied the concept of intuitionistic fuzzy sets as a generalization of fuzzy sets. There has been much progress in the study of intuitionistic fuzzy sets by many authors. Recently in (Vijayabalaji and Thillaigovindan, 2007; Vijayabalaji et al., 2007). Thillaigovindan introduced the notion of fuzzy n-inner product space and the notion of intuitionistic fuzzy n-normed linear space respectively.

In this study, we introduce the notion of Intuitionistic fuzzy n-inner product space as a generalization of fuzzy n-inner product space and prove a fixed point theorem in complete intuitionistic fuzzy ninner product space. Further this result is applied to obtain the existence and uniqueness of solution for linear volterra integral equation.

Definition 1 (Vijayabalaji et al., 2007): Let $\mathrm{n}$ be a natural number greater than 1 and $X$ be a real linear space of dimension greater than or equal to $\mathrm{n}$ and let $(\bullet$, $\bullet \mid \bullet, . . ., \cdot)$ be a real valued function on $\underbrace{X \times X \times \ldots \times X}_{n+1}=X^{n+1} \quad$ Satisfying the following conditions:

- $\quad\left(\mathrm{x}, \mathrm{x} \mid \mathrm{x}_{2}, \ldots, \mathrm{x}_{\mathrm{n}}\right) \geq 0$

- $\quad\left(\mathrm{x}, \mathrm{x} \mid \mathrm{x}_{2}, \ldots, \mathrm{x}_{\mathrm{n}}\right)=0$ if any only if $\mathrm{x}, \mathrm{x}_{2}, \ldots, \mathrm{x}_{\mathrm{n}}$ are linearly dependent

- $\left(\mathrm{x}, \mathrm{y} \mid \mathrm{x}_{2}, \ldots, \mathrm{x}_{\mathrm{n}}\right)=\left(\mathrm{y}, \mathrm{x} \mid \mathrm{x}_{2}, \ldots, \mathrm{x}_{\mathrm{n}}\right)$

- $\quad\left(\mathrm{x}, \mathrm{y} \mid \mathrm{x}_{2}, \ldots, \mathrm{x}_{\mathrm{n}}\right)$ is invariant under any permutation of $\mathrm{x}_{2}, \ldots, \mathrm{x}_{\mathrm{n}}$

- $\left(\mathrm{x}, \mathrm{x} \mid \mathrm{x}_{2}, \ldots, \mathrm{x}_{\mathrm{n}}\right)=\left(\mathrm{x}_{2}, \mathrm{x}_{2} \mid \mathrm{x}, \mathrm{x}_{3}, \ldots, \mathrm{x}_{\mathrm{n}}\right)$

- $\left(\mathrm{ax}, \mathrm{x} \mid \mathrm{x}_{2}, \ldots, \mathrm{x}_{\mathrm{n}}\right)=\mathrm{a}\left(\mathrm{x}, \mathrm{x} \mid \mathrm{x}_{2}, \ldots, \mathrm{x}_{\mathrm{n}}\right)$ for every $\mathrm{a} \in$ $\mathrm{R}$ (real)

- $\left(\mathrm{x}+x^{\prime}, \mathrm{y} \mid \mathrm{x}_{2}, \ldots, \mathrm{x}_{\mathrm{n}}\right)=\left(\mathrm{x}, \mathrm{y} \mid \mathrm{x}_{2}, \ldots, \mathrm{x}_{\mathrm{n}}\right)+\left(x^{\prime}\right.$, $\left.\mathrm{y} \mid \mathrm{x}_{2}, \ldots, \mathrm{x}_{\mathrm{n}}\right)$

Then $(\bullet, \bullet \mid \bullet, \ldots, \bullet)$ is called an n-inner product on $\mathrm{X}$ and $(\mathrm{X},(\bullet, \bullet \mid \bullet, \ldots, \bullet))$ is called an $n$-inner product space.

Definition 2 (Vijayabalaji et al., 2007): Let $n \in N$ (natural numbers) and $\mathrm{X}$ be a real linear space of

Corresponding Author: Renu Chugh, Department of Mathematics, Maharshi Dayanand University, Rohtak-124001, India 
dimension greater than or equal to $n$. A real valued function $\|\bullet, \ldots, \bullet\|$ on $\underbrace{\mathrm{X} \times \mathrm{X} \times \ldots \times \mathrm{X}}_{\mathrm{n}}=\mathrm{X}^{\mathrm{n}}$ satisfying the following four properties:

- $\left\|\mathrm{x}_{1}, \mathrm{x}_{2}, \ldots, \mathrm{x}_{\mathrm{n}}\right\|=0$ if any only if $\mathrm{x}_{1}, \mathrm{x}_{2}, \ldots, \mathrm{x}_{\mathrm{n}}$ are linearly dependent

- $\left\|\mathrm{x}_{1}, \mathrm{x}_{2}, \ldots, \mathrm{x}_{\mathrm{n}}\right\|$ is invariant under any permutation

- $\left\|\mathrm{x}_{1}, \mathrm{x}_{2}, \ldots, \mathrm{ax}_{\mathrm{n}}\right\|=|\mathrm{a}|\left\|\mathrm{x}_{1}, \mathrm{x}_{2}, \ldots, \mathrm{x}_{\mathrm{n}}\right\|$, for any a $\in \mathrm{R}$ (real)

- $\quad\left\|\mathrm{x}_{1}, \mathrm{x}_{2}, \ldots, \mathrm{x}_{\mathrm{n}-1}, \mathrm{y}+\mathrm{z}\right\| \leq\left\|\mathrm{x}_{1}, \mathrm{x}_{2}, \ldots, \mathrm{x}_{\mathrm{n}-1}, \mathrm{y}\right\|+$ $\left\|\mathrm{x}_{1}, \mathrm{x}_{2}, \ldots, \mathrm{x}_{\mathrm{n}-1}, \mathrm{z}\right\|$

Is called an n-norm on $\mathrm{X}$ and the pair $(\mathrm{X}, \| \bullet, \ldots, \bullet$ I) is called an n-normed linear space.

Remark 3 (Vijayabalaji et al., 2007): If an n-inner product space $(\mathrm{X},(\bullet, \bullet \mid \bullet, . . ., \bullet))$ is given then $\left\|\mathrm{x}_{1}, \mathrm{x}_{2}, \ldots, \mathrm{x}_{\mathrm{n}}\right\|=\sqrt{\left(\mathrm{x}_{1}, \mathrm{x}_{1} \mid \mathrm{x}_{2}, \ldots, \mathrm{x}_{\mathrm{n}}\right)}$ defines an n-norm on $X$. Further the following extension of CauchyBuniakowski inequality is also true:

$$
\mid\left(x, y \mid x_{2}, \ldots, x_{n}\right) \leq \sqrt{\left(x, x \mid x_{2}, \ldots, x_{n}\right)} \sqrt{\left(y, y \mid x_{2}, \ldots, x_{n}\right)}
$$

Definition 4: A binary operation $\Delta:[0,1] \times[0,1] \rightarrow$ $[0,1]$ is a continuous t-norm if it satisfies the following conditions:

- $\Delta$ is associative and commutative

- $\Delta$ is continuous

- a $\Delta 1=$ a for all a $\hat{I}[0,1]$

- $\mathrm{a} \Delta \mathrm{b} \leq \mathrm{c} \Delta \mathrm{d}$ whenever $\mathrm{a} \leq \mathrm{c}$ and $\mathrm{b} \leq \mathrm{d}$, for each $\mathrm{a}, \mathrm{b}$, $\mathrm{c}, \mathrm{d} \in[0,1]$

Example 5: Two typical examples of continuous tnorm are $\mathrm{a} \Delta \mathrm{b}=\mathrm{ab}$ and $\mathrm{a} \Delta \mathrm{b}=\min (\mathrm{a}, \mathrm{b})$.

Definition 6: A binary operation $\nabla:[0,1] \times[0,1] \rightarrow$ $[0,1]$ is a continuous $t$-conorm if it satisfies the following conditions:

- $\nabla$ is associative and commutative

- $\nabla$ is continuous,

- $\quad \mathrm{a} \nabla 0=$ a for all $\mathrm{a} \in[0,1]$,

- $\mathrm{a} \nabla \mathrm{b} \leq \mathrm{c} \nabla$ d whenever $\mathrm{a} \leq \mathrm{c}$ and $\mathrm{b} \leq \mathrm{d}$, for each $\mathrm{a}$, b, c, d Î $[0,1]$

Example 7: Two typical examples of continuous tconorm are $\mathrm{a} \nabla \mathrm{b}=\min (\mathrm{a}+\mathrm{b}, 1)$ and $\mathrm{a} \nabla \mathrm{b}=\max (\mathrm{a}, \mathrm{b})$.

Definition 8 (Atanassov, 1986): Let $E$ be any set. An intuitionistic fuzzy set $\mathrm{A}$ of $\mathrm{E}$ is an object of the form $\mathrm{A}$ $=\left\{\left(\mathrm{x} \mu_{\mathrm{A}}(\mathrm{x}) \gamma_{\mathrm{A}}(\mathrm{x})\right) \mid \mathrm{x} \in \mathrm{E}\right\}$, where the functions: $\mu_{\mathrm{A}}: \mathrm{E}$ $[0,1]$ and $\gamma_{\mathrm{A}} E \rightarrow[0,1] A \mathrm{~g}$ denote the degree of membership and the non-membership of the element $\mathrm{x}$ $\in \mathrm{E}$ respectively and for every $\mathrm{x} \in \mathrm{E}, 0 \leq(x)+\mu_{\mathrm{A}}(x)$ $+\gamma_{\mathrm{A}}(\mathrm{x}) \leq 1$.

Definition 9 (Vijayabalaji and Thillaigovindan, 2007): An intuitionistic fuzzy n-normed linear space is an object of the form:

$$
\left\{\begin{array}{l}
\left(X, N\left(x_{1}, x_{2}, \ldots, x_{n}, t\right), N^{\prime}\right. \\
\left.\left(x_{1}, x_{2}, \ldots, x_{n}, t\right)\right) \mid\left(x_{1}, x_{2}, \ldots, x_{n}\right) \in X^{n}
\end{array}\right\}
$$

Where $\mathrm{X}$ is a linear space over a field $\mathrm{F}, \Delta$ is a continuous t-norm, $\nabla$ is a continuous tconorm and $\mathrm{N}$, $\mathrm{N}$ ' are fuzzy sets on $\mathrm{X}^{\mathrm{n}} \times \mathrm{R}$ (R-set of real numbers) satisfying following conditions:

- $\quad \mathrm{N}\left(\mathrm{x}_{1}, \mathrm{x}_{2}, \ldots, \mathrm{x}_{\mathrm{n}}, \mathrm{t}\right)+N^{\prime}\left(\mathrm{x}_{1}, \mathrm{x}_{2}, \ldots, \mathrm{x}_{\mathrm{n}}, \mathrm{t}\right) \leq 1$

- For all $\mathrm{t} \in \mathrm{R}$ with $\mathrm{t} \leq 0, \mathrm{~N}\left(\mathrm{x}_{1}, \mathrm{x}_{2}, \ldots, \mathrm{x}_{\mathrm{n}}, \mathrm{t}\right)=0$

- For all $t \in R$ with $t>0, N\left(x_{1}, x_{2}, \ldots, x_{n}, t\right)=1$ if and only if $\mathrm{x}_{1}, \mathrm{x}_{2}, \ldots, \mathrm{x}_{\mathrm{n}}$ are linearly dependent

- $\mathrm{N}\left(\mathrm{x}_{1}, \mathrm{x}_{2}, \ldots, \mathrm{x}_{\mathrm{n}}, \mathrm{t}\right)$ is invariant under any permutation of $x_{1}, x_{2}, \ldots, x_{n}$

- For all $\mathrm{t} \in \mathrm{R}$ with $\mathrm{t}>0, \mathrm{~N}\left(\mathrm{x}_{1}, \mathrm{x}_{2}, \ldots, \mathrm{cx}_{\mathrm{n}}, \mathrm{t}\right)=\mathrm{N}$ $\left(\mathrm{x}_{1}, \mathrm{x}_{2}, \ldots, \mathrm{x}_{\mathrm{n}}, \frac{\mathrm{t}}{|\mathrm{c}|}\right)$

if $c \neq 0, c \in F($ field $)$

- For all $\mathrm{s}, \mathrm{t} \in \mathrm{R}$ :

$$
\begin{aligned}
& N\left(x_{1}, x_{2}, \ldots, x_{n}+x_{n}^{\prime}, s+t\right) \geq \Delta\left\{N\left(x_{1}, x_{2}, \ldots, x_{n}, s\right), N\right\} \\
& \left(x_{1}, x_{2}, \ldots, x_{n}^{\prime}, t\right)
\end{aligned}
$$

- $\mathrm{N}\left(\mathrm{x}_{1}, \mathrm{x}_{2}, \ldots, \mathrm{x}_{\mathrm{n}}, \mathrm{t}\right)$ is a non-decreasing function of $\mathrm{t} \in \mathrm{R}$ and:

$\lim _{t \rightarrow \infty} N\left(x_{1}, x_{2}, \ldots, x_{n}, t\right)=1$

- $\quad$ For all $\mathrm{t} \in \mathrm{R}$ with $\mathrm{t} \leq 0, N^{\prime}\left(\mathrm{x}_{1}, \mathrm{x}_{2}, \ldots, \mathrm{x}_{\mathrm{n}}, \mathrm{t}\right)=1$.

- For all $\mathrm{t} \in \mathrm{R}$ with $\mathrm{t}>0, N^{\prime}\left(\mathrm{x}_{1}, \mathrm{x}_{2}, \ldots, \mathrm{x}_{\mathrm{n}}, \mathrm{t}\right)=0$ if and only if $\mathrm{x}_{1}, \mathrm{x}_{2}, \ldots, \mathrm{x}_{\mathrm{n}}$ are linearly dependent.

- $\quad N^{\prime}\left(\mathrm{x}_{1}, \mathrm{x}_{2}, \ldots, \mathrm{x}_{\mathrm{n}}, \mathrm{t}\right)$ is invariant under any permutation of $\mathrm{x}_{1}, \mathrm{x}_{2}, \ldots, \mathrm{x}_{\mathrm{n}}$.

- $\quad$ For all $\mathrm{t} \in \mathrm{R}$ with $\mathrm{t}>0, N^{\prime}\left(\mathrm{x}_{1}, \mathrm{x}_{2}, \ldots, \mathrm{cx}_{\mathrm{n}}, \mathrm{t}\right)=N^{\prime}$ $\left(\mathrm{x}_{1}, \mathrm{x}_{2}, \ldots, \mathrm{x}_{\mathrm{n}}, \frac{\mathrm{t}}{|\mathrm{c}|}\right)$ :

if $c \neq 0, c \in F($ field) 
- For all $\mathrm{s}, \mathrm{t} \in \mathrm{R}$ :

$$
\begin{aligned}
& N^{\prime}\left(x_{1}, x_{2}, \ldots, x_{n}+x_{n}^{\prime}, s+t\right) \leq \nabla \\
& \left\{N^{\prime}\left(x_{1}, x_{2}, \ldots, x_{n}, s\right), N^{\prime}\left(x_{1}, x_{2}, ., x_{n}, t\right)\right\}
\end{aligned}
$$

$N^{\prime}\left(x_{1}, x_{2}, \ldots, x_{n}, t\right)$ is a non-increasing function of $t \in$ $\mathrm{R}$ and:

$$
\lim _{t \rightarrow \infty} N^{\prime}\left(x_{1}, x_{2}, \ldots, x_{n}, t\right)=0
$$

Definition 10: Let $X$ be a linear space over a field $F$. A fuzzy subset $\mathrm{J}: \mathrm{X}^{\mathrm{n}+1} \times \mathrm{R}$ (Real numbers) is called a fuzzy $\mathrm{n}$ - inner poduct on $\mathrm{X}$ if and only if:

- $\quad$ For all $\mathrm{t} \in \mathrm{R}$ with $\mathrm{t} \leq 0, \mathrm{~J}\left(\mathrm{x}, \mathrm{x} \mid \mathrm{x}_{1}, \ldots, \mathrm{x}_{\mathrm{n}-1}, \mathrm{t}\right)=0$

- For all $t \in R$ with $t>0, J\left(x, x \mid x_{1}, \ldots, x_{n-1}, t\right)=1$ if and only if $\mathrm{x}, \mathrm{x}_{1}, \ldots, \mathrm{x}_{\mathrm{n}-1}$ are linearly dependent

- $\quad$ For all $\mathrm{t}>0, \mathrm{~J}\left(\mathrm{x}, \mathrm{y} \mid \mathrm{x}_{1}, \ldots, \mathrm{x}_{\mathrm{n}-1}, \mathrm{t}\right)=\mathrm{J}\left(\mathrm{y}, \mathrm{x} \mid \mathrm{x}_{1}, \ldots\right.$ $\left., \mathrm{x}_{\mathrm{n}-1}, \mathrm{t}\right)$

- $\mathrm{J}\left(\mathrm{x}, \mathrm{y} \mid \mathrm{x}_{1}, \ldots, \mathrm{x}_{\mathrm{n}-1}, \mathrm{t}\right)$ is invariant under any permutation of $\mathrm{x}_{1}, \ldots, \mathrm{x}_{\mathrm{n}-1}$

- $\quad$ For all $\mathrm{t}>0, \mathrm{~J}\left(\mathrm{x}, \mathrm{x} \mid \mathrm{x}_{1}, \ldots, \mathrm{x}_{\mathrm{n}-1}, \mathrm{t}\right)=\mathrm{J}\left(\mathrm{x}_{1}, \mathrm{x}_{1} \mid \mathrm{x}, \mathrm{x}_{2}\right.$, . $\left.\ldots, \mathrm{x}_{\mathrm{n}-1}, \mathrm{t}\right)$

- $\quad$ For all $\mathrm{t}>0, \mathrm{~J}\left(\mathrm{ax}, \mathrm{bx} \mid \mathrm{x}_{1}, \ldots, \mathrm{x}_{\mathrm{n}-1}, \mathrm{t}\right)=\mathrm{J}\left(\mathrm{x}, \mathrm{x} \mid \mathrm{x}_{1}, \ldots\right.$ $\left.., \mathrm{x}_{\mathrm{n}-1}, \frac{\mathrm{t}}{|\mathrm{ab}|}\right), \mathrm{a}, \mathrm{b} \in \mathrm{R}($ real $)$

- For all $\mathrm{s}, \mathrm{t} \in \mathrm{R}$ :

$$
\begin{aligned}
& J\left(x, y \mid x_{1}, \ldots, x_{n-1}, \sqrt{t s}\right) \geq \min \\
& \left\{J\left(x \mid x_{1} . t\right), J\left(y, y Z\left(x_{1}, \ldots, x_{n-1}, t\right)\right\}\right.
\end{aligned}
$$

$\mathrm{J}\left(\mathrm{x}+x \phi, \mathrm{y} \mid \mathrm{x}_{1}, \ldots, \mathrm{x}_{\mathrm{n}-1}, \mathrm{t}+\mathrm{s}\right) \_\min \left\{\mathrm{J}\left(\mathrm{x}, \mathrm{y} \mid \mathrm{x}_{1}, \ldots, \mathrm{x}_{\mathrm{n}-1}\right.\right.$, $\left.\mathrm{t}), \mathrm{J}\left(x \notin, \mathrm{y} \mid \mathrm{x}_{1}, \ldots, \mathrm{x}_{\mathrm{n}-1}, \mathrm{~s}\right)\right\}$.

- For all $s, t \in R$ with $s>0, t>0$ :

$$
\begin{aligned}
& \mathrm{J}\left(\mathrm{x}, \mathrm{y} \mid \mathrm{x}_{1}, \ldots, \mathrm{x}_{\mathrm{n}-1}, \sqrt{\mathrm{ts}}\right) \geq \min \left\{\mathrm{J}\left(\mathrm{x}, \mathrm{x} \mid \mathrm{x}_{1}, \ldots, \mathrm{x}_{\mathrm{n}-1}, \mathrm{t}\right),\right. \\
& \left.\mathrm{J}\left(\mathrm{y}, \mathrm{y} \mid \mathrm{x}_{1}, \ldots, \mathrm{x}_{\mathrm{n}-1}, \mathrm{t}\right)\right\}
\end{aligned}
$$

- $\mathrm{J}\left(\mathrm{x}, \mathrm{y} \mid \mathrm{x}_{1}, \ldots, \mathrm{x}_{\mathrm{n}-1}, \mathrm{t}\right)$ is a non-decreasing function of $\mathrm{t} \in \mathrm{R}$ and:

$$
\lim _{t \rightarrow \infty} J\left(x, y \mid x_{1}, \ldots, x_{n-1}, t\right)=1
$$

Then $(X, J)$ is called a fuzzy $n$-inner product space or in short f-n-IPS.

Intuitionistic fuzzy n-inner product space: We introduce the notion of intuitionistic fuzzy n-inner product space as a generalization of Definition 10 as follows.
Definition 11: An intuitionistic fuzzy n-inner poduct space is an object of the form:

$$
A=\left\{\begin{array}{l}
\left(X, J\left(x, y \mid x_{1}, x_{2}, \ldots, x_{n-1}, t\right)\right. \\
\left.J^{\prime}\left(x, y \mid x_{1}, x_{2}, \ldots, x_{n-1}, t\right)\right) \mid\left(x, y, x_{1}, x_{2}, \ldots, x_{n-1}\right) \in X^{n+1}
\end{array}\right\}
$$

where, $\mathrm{X}$ is a linear space over a field $\mathrm{F}, \Delta$ is a continuous t-norm, $\nabla$ is a continuous tconorm and $\mathbf{J}, \mathrm{J}$ ' are fuzzy sets on $\mathrm{X}^{\mathrm{n}+1} \times \mathrm{R}$ (R-set of real numbers) satisfying following conditions:

- $\quad \mathrm{J}\left(\mathrm{x}, \mathrm{y} \mid \mathrm{x}_{1}, \ldots, \mathrm{x}_{\mathrm{n}-1}, \mathrm{t}\right)+\mathrm{J}^{\prime}\left(\mathrm{x}, \mathrm{y} \mid \mathrm{x}_{1}, \ldots, \mathrm{x}_{\mathrm{n}-1}, \mathrm{t}\right) \leq 1$

- For all $\mathrm{t} \in \mathrm{R}$ with $\mathrm{t} \leq 0, \mathrm{~J}\left(\mathrm{x}, \mathrm{x} \mid \mathrm{x}_{1}, \ldots, \mathrm{x}_{\mathrm{n}-1}, \mathrm{t}\right)=0$ (or $\theta$, the null vector)

- For all $\mathrm{t} \in \mathrm{R}$ with $\mathrm{t}>0, \mathrm{~J}\left(\mathrm{x}, \mathrm{x} \mid \mathrm{x}_{1}, \ldots, \mathrm{x}_{\mathrm{n}-1}, \mathrm{t}\right)=1$ if and only if $\mathrm{x}, \mathrm{x}_{1}, \ldots, \mathrm{x}_{\mathrm{n}-1}$ are linearly dependent

- $\quad$ For all $\mathrm{t}>0, \mathrm{~J}\left(\mathrm{x}, \mathrm{y} \mid \mathrm{x}_{1}, \ldots, \mathrm{x}_{\mathrm{n}-1}, \mathrm{t}\right)=\mathrm{J}\left(\mathrm{y}, \mathrm{x} \mid \mathrm{x}_{1}, \ldots\right.$, $\left.\mathrm{x}_{\mathrm{n}-1}, \mathrm{t}\right)$

- $\mathrm{J}\left(\mathrm{x}, \mathrm{y} \mid \mathrm{x}_{1}, \ldots, \mathrm{x}_{\mathrm{n}-1}, \mathrm{t}\right)$ is invariant under any permutation of $\mathrm{x}_{1}, \ldots, \mathrm{x}_{\mathrm{n}-1}$

- $\quad$ For all $\mathrm{t}>0, \mathrm{~J}\left(\mathrm{x}, \mathrm{x} \mid \mathrm{x}_{1}, \ldots, \mathrm{x}_{\mathrm{n}-1}, \mathrm{t}\right)=\mathrm{J}\left(\mathrm{x}_{1}, \mathrm{x}_{1} \mid \mathrm{x}, \mathrm{x}_{2}\right.$, . $\left.\ldots, \mathrm{x}_{\mathrm{n}-1}, \mathrm{t}\right)$

- $\quad$ For all $\mathrm{t}>0, \mathrm{~J}\left(\mathrm{ax}, \mathrm{bx} \mid \mathrm{x}_{1}, \ldots, \mathrm{x}_{\mathrm{n}-1}, \mathrm{t}\right)=\mathrm{J}\left(\mathrm{x}, \mathrm{x} \mid \mathrm{x}_{1}, \ldots\right.$ . , $\left.\mathrm{x}_{\mathrm{n}-1}, \frac{\mathrm{t}}{|\mathrm{ab}|}\right) \mathrm{a}, \mathrm{b} \in \mathrm{R}($ real $)$

- For all $\mathrm{s}, \mathrm{t} \in \mathrm{R}$ :

$$
\begin{aligned}
& J\left(x+x^{\prime}, y \mid x_{1}, \ldots, x_{n-1}, t+s\right) \geq \Delta \\
& \left\{J\left(x, y \mid x_{1}, \ldots, x_{n-1}, t\right)\left(x_{1}, \ldots, x_{n-1}, s\right)\right\}
\end{aligned}
$$

- $\quad$ For all $\mathrm{s}, \mathrm{t} \in \mathrm{R}$ with $\mathrm{s}>0, \mathrm{t}>0$ :

$$
\begin{aligned}
& \mathrm{J}\left(\mathrm{x}, \mathrm{y} \mid \mathrm{x}_{1}, \ldots, \mathrm{x}_{\mathrm{n}-1}, \sqrt{\mathrm{ts}}\right) \geq \Delta\left\{\mathrm{J}\left(\mathrm{x}, \mathrm{x} \mid \mathrm{x}_{1}, \ldots, \mathrm{x}_{\mathrm{n}-1}, \mathrm{t}\right),\right. \\
& \left.\mathrm{J}\left(\mathrm{y}, \mathrm{y} \mid \mathrm{x}_{1}, \ldots, \mathrm{x}_{\mathrm{n}-1}, \mathrm{t}\right)\right\}
\end{aligned}
$$

- $\mathrm{J}\left(\mathrm{x}, \mathrm{y} \mid \mathrm{x}_{1}, \ldots, \mathrm{x}_{\mathrm{n}-1}, \mathrm{t}\right)$ is a non-decreasing function of $\mathrm{t} \in \mathrm{R}$ and:

$\lim _{t \rightarrow \infty} J\left(x, y \mid x_{1}, \ldots, x_{n-1}, t\right)=1$

- For all $t \in R$ with $t \leq 0, J^{\prime}\left(x, x \mid x_{1}, \ldots, x_{n-1}, t\right)=1$

- For all $t \in R$ with $t>0, J^{\prime}\left(x, x \mid x_{1}, \ldots, x_{n-1}, t\right)=0$ if and only if $\mathrm{x}, \mathrm{x}_{1}, \ldots, \mathrm{x}_{\mathrm{n}-1}$ are linearly dependent

- $\quad$ For all $\mathrm{t}>0, \mathrm{~J}^{\prime}\left(\mathrm{x}, \mathrm{y} \mid \mathrm{x}_{1}, \ldots, \mathrm{x}_{\mathrm{n}-1}, \mathrm{t}\right)=\mathrm{J}^{\prime}\left(\mathrm{y}, \mathrm{x} \mid \mathrm{x}_{1}, \ldots\right.$ $\left., \mathrm{x}_{\mathrm{n}-1}, \mathrm{t}\right)$

- $\mathrm{J}^{\prime}\left(\mathrm{x}, \mathrm{y} \mid \mathrm{x}_{1}, \ldots, \mathrm{x}_{\mathrm{n}-1}, \mathrm{t}\right)$ is invariant under any permutation of $\mathrm{x}_{1}, \ldots, \mathrm{x}_{\mathrm{n}-1}$

- For all $\mathrm{t}>0, \mathrm{~J} \mathrm{~J}^{\prime}\left(\mathrm{x}, \mathrm{x} \mid \mathrm{x}_{1}, \ldots, \mathrm{x}_{\mathrm{n}-1}, \mathrm{t}\right)=\mathrm{J}^{\prime}\left(\mathrm{x}_{1}, \mathrm{x}_{1} \mid \mathrm{x}\right.$, $\left.\mathrm{x}_{2}, \ldots, \mathrm{x}_{\mathrm{n}-1}, \mathrm{t}\right)$ 
- For all $\mathrm{t}>0, \mathrm{~J}^{\prime}\left(\mathrm{ax}, \mathrm{bx} \mid \mathrm{x}_{1}, \ldots, \mathrm{x}_{\mathrm{n}-\mathrm{l}}, \mathrm{t}\right)=\mathrm{J}^{\prime}\left(\mathrm{x}, \mathrm{x} \mid \mathrm{x}_{1}\right.$, $\left.\ldots, x_{n-1}, \frac{t}{|a b|}\right) a, b \in R$

- For all $\mathrm{s}, \mathrm{t} \in \mathrm{R}$ :

$$
\begin{aligned}
& J^{\prime}\left(x+x^{\prime}, y \mid x_{1}, \ldots, x_{n-1}, t+s\right) \leq \nabla\left\{x, y \mid x_{1}, \ldots, x_{n-1}, t\right\} \\
& \left.J^{\prime}\left(x^{\prime}, y \mid x_{1}, \ldots, x_{n-1}, s\right)\right\}
\end{aligned}
$$

- $\quad$ For all $\mathrm{s}, \mathrm{t} \in \mathrm{R}$ with $\mathrm{s}>0, \mathrm{t}>0$ :

$$
\begin{aligned}
& \mathrm{J}^{\prime}\left(\mathrm{x}, \mathrm{y} \mid \mathrm{x}_{1}, \ldots, \mathrm{x}_{\mathrm{n}-1}, \sqrt{\mathrm{ts}}\right) \leq \nabla\left\{\mathrm{J}^{\prime}\left(\mathrm{x}, \mathrm{x} \mid \mathrm{x}_{1}, \ldots ., \mathrm{x}_{\mathrm{n}-1}, \mathrm{t}\right),\right. \\
& \left.\mathrm{J}^{\prime}\left(\mathrm{x}, \mathrm{y} \mid \mathrm{x}_{1}, \ldots, \mathrm{x}_{\mathrm{n}-1}, \mathrm{~s}\right)\right\}
\end{aligned}
$$

$J^{\prime}\left(x, y \mid x_{1}, \ldots, x_{n-1}, t\right)$ is a non-increasing function of $\mathrm{t} \in \mathrm{R}$ and:

$$
\lim _{\mathrm{t} \rightarrow \infty} \mathrm{J}^{\prime}\left(\mathrm{x}, \mathrm{y} \mid \mathrm{x}_{1}, \ldots, \mathrm{x}_{\mathrm{n}-1}, \mathrm{t}\right)=0
$$

Then $\left(\mathrm{X}, \mathrm{J}, J^{\prime}, \mathrm{D}, \tilde{\mathrm{N}}\right)$ is called an intuitionistic fuzzy n-inner product space or in short IF-n-IPS.

Example 12: Let $(\mathrm{X},(\bullet, \bullet \mid \bullet, \ldots, \bullet))$ be an $\mathrm{n}$ - inner product space. Define a $\nabla \mathrm{b}=\min (\mathrm{a}, \mathrm{b})$ and $\mathrm{a} \in \mathrm{b}=$ $\max (a, b)$, for all $a, b \in[0,1]$ :

$$
\begin{aligned}
& J\left(x, y \mid x_{1}, \ldots, x_{n-1}, t\right)=\left\{\frac{t}{t+\mid\left(x, y \mid x_{1}, \ldots, x_{n-1}\right)}\right. \\
& \text { when } t>0, t \in R,\left(x, y \mid x_{1}, \ldots, x_{n-1}\right) \in X^{n+1} \\
& 0, \text { when } t \mid \leq 0
\end{aligned}
$$

And:

$$
\begin{aligned}
& J^{\prime}\left(x, y \mid x_{1}, \ldots, x_{n-1}, t\right)=\left\{\frac{\left|\left(x, y \mid x_{1}, \ldots, x_{n-1}\right)\right|}{t+\mid\left(x, y \mid x_{1}, \ldots, x_{n-1}\right)}\right. \\
& \text { when } t>0, t \in R,\left(x, y \mid x_{1}, \ldots, x_{n-1}\right) \in X^{n+1} \\
& 1 \text {, when } t \leq 0
\end{aligned}
$$

Then $\left(\mathrm{X}, \mathrm{J}, \mathrm{J}^{\prime}, \Delta, \nabla\right)$ is an IF-n-IPS.

\section{Proof:}

- $\quad$ Clearly $J\left(x, y \mid x_{1}, . ., x_{n-1}, t\right)+J^{\prime}\left(x, y \mid x_{1}, \ldots, x_{n-1}, t\right) \leq 1$

- For all $t \in R$ with $t \leq 0$ we have by our definition, $J$ $\left(\mathrm{x}, \mathrm{x} \mid \mathrm{x}_{1}, \ldots, \mathrm{x}_{\mathrm{n}-1}, \mathrm{t}\right)=0$

- For all $\mathrm{t} \in \mathrm{R}$ with $\mathrm{t}>0$ we have, $\mathrm{J}\left(\mathrm{x}, \mathrm{x} \mid \mathrm{x}_{1}, \ldots, \mathrm{x}_{\mathrm{n}-1}, \mathrm{t}\right)$ $=1$ :

$$
\frac{t}{t+\left(x, x \mid x_{1}, ., x_{n-1}\right)}=1 \Leftrightarrow\left|\left(x, x \mid x_{1}, \ldots, x_{n-1}\right)\right|=0
$$

$\left|\left(\mathrm{x}, \mathrm{x} \mid \mathrm{x}_{1}, \ldots, \mathrm{x}_{\mathrm{n}-1}\right)\right|=0 \Leftrightarrow \mathrm{x}, \mid \mathrm{x}_{1}, \ldots, \mathrm{x}_{\mathrm{n}-1}$ are linearly dependent.

- For all $\mathrm{t}>0$ :

$$
\begin{aligned}
& J\left(x, y \mid x_{1}, \ldots, x_{n-1}, t\right)=\frac{t}{t+\left|\left(x, y \mid x_{1}, \ldots, x_{n-1}\right)\right|} \\
& =\frac{t}{t+\left|\left(y, x \mid x_{1}, \ldots, x_{n-1}\right)\right|}=J\left(y, x \mid x_{1}, \ldots, x_{n-1}, t\right)
\end{aligned}
$$

As $\left(\mathrm{x}, \mathrm{x} \mid \mathrm{x}_{1} \ldots \mathrm{x}_{\mathrm{n}-1}, \mathrm{t}\right)$ is invariant under any permutation of $x_{1}, \ldots, x_{n-1}$.

We have $\mathrm{J}\left(\mathrm{x}, \mathrm{y} \mid \mathrm{x}_{1} \ldots \mathrm{x}_{\mathrm{n}-1}, \mathrm{t}\right)$ is invariant under any permutation of $\mathrm{x}_{1}, \ldots, \mathrm{x}_{\mathrm{n}-1}$.

For all $\mathrm{t}>0$ :

$$
\begin{aligned}
& J\left(x, x \mid x_{1}, \ldots, x_{n-1}, t\right)=\frac{t}{t+\left|\left(x_{1}, x_{1} \mid x, \ldots, x_{n-1}\right)\right|} \\
& =\frac{t}{t+\left|\left(x_{1}, x_{1} \mid x, \ldots, x_{n-1}\right)\right|}=J\left(x_{1}, x_{1} \mid x, \ldots, x_{n-1}, t\right)
\end{aligned}
$$

For all $\mathrm{t}>0$ :

$$
\begin{aligned}
J\left(x, x \mid x_{1}, \ldots, x_{n-1}, \frac{t}{|a b|}\right)= & \frac{\frac{t}{|a b|}}{\frac{t}{|a b|}+\left|\left(x, x \mid x_{1}, \ldots, x_{n-1}\right)\right|} \\
& =\frac{t}{|t+| a b \|\left(x, x \mid x_{1}, \ldots, x_{n-1}\right) \mid} \\
& =\frac{t}{t+\left|\left(a x, b x \mid x_{1}, \ldots . x_{n-1}\right)\right|} \\
& =J\left(a x, b x \mid x_{1}, \ldots, x_{n-1}, t\right)
\end{aligned}
$$

If (a) $\mathrm{s}+\mathrm{t}<0$ (b) $\mathrm{s}=\mathrm{t}=0$ (c) $\mathrm{s}+\mathrm{t}>0$; $\mathrm{s}>0, \mathrm{t}<0$; $\mathrm{s}\langle 0, \mathrm{t}>0$, Then the above relation is obvious. If (d) $\mathrm{s}>$ $0, t>0, s+t>0$. Then Without loss of generality assume that:

$$
\begin{aligned}
& J\left(x, y \mid x_{1}, \ldots, x_{n-1}, t\right) \leq J\left(x^{\prime}, y \mid x_{1}, \ldots, x_{n-1}, s\right) \\
& \frac{t}{t+\left|\left(x, y \mid x_{1}, \ldots, x_{n-1}\right)\right|} \leq \frac{s}{s+\left|\left(x, y \mid x_{1}, \ldots, x_{n-1}\right)\right|} \\
& \frac{t+\left|\left(x, y \mid x_{1}, \ldots, x_{n-1}\right)\right|}{t} \geq \frac{s+\left|\left(x^{\prime}, y \mid x_{1}, \ldots, x_{n-1}\right)\right|}{s} \\
& 1+\frac{\left|\left(x, y \mid x_{1}, \ldots, x_{n-1}\right)\right|}{t} \geq 1+\frac{\left|\left(x^{\prime}, y|| x_{1}, \ldots, x_{n-1}\right)\right|}{s} \\
& \frac{\left|\left(x, y \mid x_{1}, \ldots, x_{n-1}\right)\right|}{t} \geq \frac{\left|\left(x^{\prime}, y \mid x_{1}, \ldots, x_{n-1}\right)\right|}{s} \\
& \frac{\mid\left(x, y \mid x_{1}, \ldots, x_{n-1}\right)}{t} \geq\left|\left(x^{\prime}, y \mid x_{1}, \ldots, x_{n-1}\right)\right|
\end{aligned}
$$




$$
\begin{aligned}
& \left|\left(x, y \mid x_{1}, \ldots, x_{n-1}\right)\right|+\frac{\mid\left(x, y \mid x_{1}, \ldots, x_{n-1}\right)}{t} \geq \\
& \left|\left(x, y \mid x_{1}, \ldots, x_{n-1}\right)\right|+\left|\left(x^{\prime}, y \mid x_{1}, \ldots, x_{n-1}\right)\right| \\
& \left(1+\frac{s}{t}\right) Z\left(x, y \mid x_{1}, \ldots, x_{n-1}\right)|\geq|\left(x+x^{\prime}, y \mid x_{1}, \ldots, x_{n-1}\right) \mid \\
& \frac{s+t}{t}\left|\left(x, y \mid x_{1}, \ldots, x_{n-1}\right)\right| \geq\left|\left(x+x^{\prime}, y \mid x_{1}, \ldots, x_{n-1}\right)\right| \\
& \frac{\mid\left(x, y \mid x_{1}, \ldots, x_{n-1}\right)}{t} \geq \frac{\left|\left(x+x^{\prime}, y \mid x_{1}, \ldots, x_{n-1}\right)\right|}{s+t} \\
& 1+\frac{\left|\left(x, y \mid x_{1}, \ldots, x_{n-1}\right)\right|}{t} \geq 1+\frac{\left|\left(x+x^{\prime}, y \mid x_{1}, \ldots, x_{n-1}\right)\right|}{s+t} \\
& \frac{t+\mid\left(x, y \mid x_{1}, \ldots, x_{n-1}\right)}{t} \geq \frac{s+t+\left|\left(x+x^{\prime}, y \mid x_{1}, \ldots, x_{n-1}\right)\right|}{s+t} \\
& \frac{t}{t+\left|\left(x, y \mid x_{1}, \ldots, x_{n-1}\right)\right|} \leq \frac{s+t}{s+t+\left|\left(x, y \mid x_{1}, \ldots, x_{n-1}\right)\right|} \\
& \min \left\{J\left(x, y \mid x_{1}, \ldots, x_{n-1}, t\right), J\left(x x^{\prime} y \mid x_{1}, \ldots, x_{n-1}, s\right)\right\} \\
& \leq J\left(x+x^{\prime}, y \mid x_{1}, \ldots, x_{n-1}, s+t\right)
\end{aligned}
$$

Without loss of generality assume that:

$\mathrm{J}\left(\mathrm{x}, \mathrm{x} \mid \mathrm{x}_{1}, \ldots, \mathrm{x}_{\mathrm{n}-1}, \mathrm{t}\right) \leq \mathrm{J}\left(\mathrm{y}, \mathrm{y} \mid \mathrm{x}_{1}, \ldots, \mathrm{x}_{\mathrm{n}-1}, \mathrm{~s}\right)$ for all $\mathrm{s}, \mathrm{t} \in$ with $\mathrm{s}>0, \mathrm{t}>0$ :

$$
\begin{gathered}
\frac{J\left(x, x \mid x_{1}, \ldots, x_{n-1}\right) \leq J\left(y, y \mid x_{1}, \ldots, x_{n-1}\right)}{t+\left|\left(x, x \mid x_{1}, \ldots, x_{n-1}\right)\right|} \leq \frac{s}{s+\left|\left(y, y \mid x_{1}, \ldots, x_{n-1}\right)\right|} \\
\frac{t+\left|\left(x, x \mid x_{1}, \ldots, x_{n-1}\right)\right|}{t} \geq \frac{s+\left|\left(y, y \mid x_{1}, \ldots, x_{n-1}\right)\right|}{s} \\
1+\frac{\left|\left(x, x \mid x_{1}, \ldots, x_{n-1}\right)\right|}{t} \geq 1+\frac{\left|\left(y, y \mid x_{1}, \ldots, x_{n-1}\right)\right|}{s} \\
\quad \geq\left|\left(x, x \mid x_{1}, \ldots, x_{n-1}\right)\right|\left|\left(y, y \mid x_{1}, \ldots, x_{n-1}\right)\right| \\
\frac{\left|\left(x, x \mid x_{1}, \ldots, x_{n-1}\right)\right|}{t} \geq \frac{\left|\left(y, y \mid x_{1}, \ldots, x_{n-1}\right)\right|}{t} \\
\frac{s\left|\left(x, x \mid x_{1}, \ldots, x_{n-1}\right)\right|}{t} \geq\left|\left(y, y \mid x_{1}, \ldots, x_{n-1}\right)\right|
\end{gathered}
$$

\section{By Remark:}

$$
\begin{aligned}
& \frac{\mathrm{s}\left|\left(\mathrm{x}, \mathrm{x} \mid \mathrm{x}_{1}, \ldots, \mathrm{x}_{\mathrm{n}-1}\right)\right|^{2}}{\mathrm{t}} \geq\left|\left(\mathrm{x}, \mathrm{y} \mid \mathrm{x}_{1}, \ldots, \mathrm{x}_{\mathrm{n}-1}\right)\right|^{2} \\
& \frac{\left|\left(\mathrm{x}, \mathrm{x} \mid \mathrm{x}_{1}, \ldots, \mathrm{x}_{\mathrm{n}-1}\right)\right|^{2} \mathrm{~s}}{\mathrm{t}^{2}} \geq\left|\left(\mathrm{x}, \mathrm{y} \mid \mathrm{x}_{1}, \ldots, \mathrm{x}_{\mathrm{n}-1}\right)\right|^{2} \\
& \frac{\left|\left(\mathrm{x}, \mathrm{x} \mid \mathrm{x}_{1}, \ldots, \mathrm{x}_{\mathrm{n}-1}\right)\right|^{2}}{\mathrm{t}^{2}} \geq \frac{\left|\left(\mathrm{x}, \mathrm{y} \mid \mathrm{x}_{1}, \ldots, \mathrm{x}_{\mathrm{n}-1}\right)\right|^{2}}{\mathrm{st}}
\end{aligned}
$$

Taking square root on both sides:

$$
\begin{aligned}
& \frac{\left|\left(\mathrm{x}, \mathrm{x} \mid \mathrm{x}_{1}, \ldots, \mathrm{x}_{\mathrm{n}-1}\right)\right|}{\mathrm{t}} \geq \frac{\left|\left(\mathrm{x}, \mathrm{y} \mid \mathrm{x}_{1}, \ldots, \mathrm{x}_{\mathrm{n}-1}\right)\right|}{\sqrt{\mathrm{st}}} \\
& \frac{1+\frac{\left|\left(\mathrm{x}, \mathrm{x} \mid \mathrm{x}_{1}, \ldots, \mathrm{x}_{\mathrm{n}-1}\right)\right|}{\mathrm{t}} \geq 1+\frac{\left|\left(\mathrm{x}, \mathrm{y} \mid \mathrm{x}_{1}, \ldots, \mathrm{x}_{\mathrm{n}-1}\right)\right|}{\sqrt{\mathrm{st}}}}{\mathrm{t}} \\
& \frac{\left.\mathrm{t} \mathrm{x}, \mathrm{x} \mid \mathrm{x}_{1}, \ldots, \mathrm{x}_{\mathrm{n}-1}\right) \mid}{\mathrm{t}+\left|\left(\mathrm{x}, \mathrm{x} \mid \mathrm{x}_{1}, \ldots, \mathrm{x}_{\mathrm{n}-1}\right)\right|} \leq \frac{\sqrt{\mathrm{st}+\left|\left(\mathrm{x}, \mathrm{y} \mid \mathrm{x}_{1}, \ldots, \mathrm{x}_{\mathrm{n}-1}\right)\right|}}{\sqrt{\mathrm{st}}} \\
& \frac{\mathrm{t}}{\mathrm{t}+\mid\left(\mathrm{x}, \mathrm{x} \mid \mathrm{x}_{1}, \ldots, \mathrm{x}_{\mathrm{n}-1}, \mathrm{t}\right)} \leq \frac{\sqrt{\mathrm{st}}}{\sqrt{\mathrm{st}}+\left|\left(\mathrm{x}, \mathrm{y} \mid \mathrm{x}_{1}, \ldots, \mathrm{x}_{\mathrm{n}-1}\right)\right|} \\
& \min \left\{\mathrm{J}\left(\mathrm{x}, \mathrm{x}_{1}, \ldots, \mathrm{x}_{1}, \ldots, \mathrm{x}_{\mathrm{n}-1}, \mathrm{t}\right), \mathrm{J}\left(\mathrm{y}, \mathrm{y} \mid \mathrm{x}_{1}, \ldots, \mathrm{x}_{\mathrm{n}-1}, \mathrm{~s}\right)\right\} \\
& \leq \mathrm{J}\left(\mathrm{x}, \mathrm{y} \mid \mathrm{x}_{1}, \ldots, \mathrm{x}_{\mathrm{n}-1}, \sqrt{\mathrm{ts}}\right)
\end{aligned}
$$

For all $\mathrm{t}_{1}<\mathrm{t}_{2} \leq 0$ then, by our definition:

$$
J\left(x, y \mid x_{1}, \ldots, x_{n-1}\right)\left|=J\left(x, y \mid x_{1}, \ldots, x_{n-1}\right)\right|=0 .
$$

Suppose $t_{2}>t_{1}>0$ then:

$$
\begin{aligned}
& \frac{t_{2}}{t_{2}+\left|\left(x, y \mid x_{1}, \ldots, x_{n-1}\right)\right|}-\frac{t_{1}}{t_{1}+\left|\left(x, y \mid x_{1}, \ldots, x_{n-1}\right)\right|} \\
& =\frac{\left|\left(x, y \mid x_{1}, \ldots, x_{n-1}\right)\right|\left(t_{2}-t_{1}\right)}{\left(t_{2}+\left|\left(x, y \mid x_{1}, \ldots, x_{n-1}\right)\right|\right)\left(t_{1}+\left|\left(x, y \mid x_{1}, \ldots, x_{n-1}\right)\right|\right)} \\
& \geq 0, \text { for all }\left(x, y \mid x_{1}, \ldots, x_{n-1}\right) \in X^{n+1} \\
& \frac{t_{2}}{t_{2}+\left|\left(x, y \mid x_{1}, \ldots, x_{n-1}\right)\right|} \geq \frac{t_{1}}{t_{1}+\left|\left(x, y \mid x_{1}, \ldots, x_{n-1}\right)\right|} \\
& J\left(x, y \mid x_{1}, \ldots, x_{n-1} t_{2}\right) \geq J\left(x, y \mid x_{1}, \ldots, x_{n-1}, t_{1}\right)
\end{aligned}
$$

Thus $\mathbf{J}\left(\mathrm{x} \quad \mathrm{y} \mid \mathrm{x}_{1}, \ldots, \mathrm{x}_{\mathrm{n}-1}, \mathrm{t}\right)$ is non-decreasing function. Also:

$$
\begin{aligned}
& \lim _{t \rightarrow \infty} J\left(x, y \mid x_{1}, \ldots, x_{n-1}\right)=\lim _{t \rightarrow \infty} \frac{t}{t+\left|\left(x, y \mid x_{1}, \ldots, x_{n-1}\right)\right|} \\
& =\lim _{t \rightarrow \infty} \frac{t}{t\left(1+\frac{1}{t}|x, y| x_{1}, \ldots, x_{n-1}\right) \mid}=1 .
\end{aligned}
$$

For all $\mathrm{t} \in \mathrm{R}$ with $\mathrm{t} \leq 0$ we have by our definition:

$$
\mathrm{J}^{\prime}\left(\mathrm{x}, \mathrm{x} \mid \mathrm{x}_{1}, \ldots, \mathrm{x}_{\mathrm{n}-1}\right)=1
$$

For all $\mathrm{t} \in \mathrm{R}$ with $t>0$ we have:

$$
\begin{aligned}
& J^{\prime}\left(\mathrm{x}, \mathrm{x} \mid \mathrm{x}_{1}, \ldots, \mathrm{x}_{\mathrm{n}-1}, \mathrm{t}\right)=0 \\
& \frac{\left|\left(\mathrm{x}, \mathrm{x} \mid \mathrm{x}_{1}, \ldots, \mathrm{x}_{\mathrm{n}-1}\right)\right|}{\mathrm{t}+\left|\left(\mathrm{x}, \mathrm{x} \mid \mathrm{x}_{1}, \ldots, \mathrm{x}_{\mathrm{n}-1}\right)\right|}=0\left|\left(\mathrm{x}, \mathrm{x} \mid \mathrm{x}_{1}, \ldots, \mathrm{x}_{\mathrm{n}-1}\right)\right|=0 \\
& \left|\left(\mathrm{x}, \mathrm{x} \mid \mathrm{x}_{1}, \ldots, \mathrm{x}_{\mathrm{n}-1}\right)\right|=0 \mathrm{x} \cdot \mathrm{x}_{1}, \ldots, \mathrm{x}_{\mathrm{n}-1} \\
& \text { are linearly dependent }
\end{aligned}
$$


For all $\mathrm{t}>0$ :

$$
\begin{aligned}
J^{\prime}\left(x, y \mid x_{1}, \ldots, x_{n-1}, t\right)= & \frac{\left|\left(x, y \mid x_{1}, \ldots, x_{n-1}\right)\right|}{t+\left|\left(x, y \mid x_{1}, \ldots, x_{n-1}\right)\right|} \\
& =\frac{\left|\left(y, x \mid x_{1}, \ldots, x_{n-1}\right)\right|}{t+\left|\left(y, x \mid x_{1}, \ldots, x_{n-1}\right)\right|} \\
& =J^{\prime}\left(y, x \mid x_{1}, \ldots, x_{n-1}, t\right) .
\end{aligned}
$$

As $\left(\mathrm{x}, \mathrm{x} \mid \mathrm{x}_{1}, \ldots, \mathrm{x}_{\mathrm{n}-1}, \mathrm{t}\right)$ is invariant under any permutation of $\mathrm{x}_{1}, \ldots, \mathrm{x}_{\mathrm{n}-1}$.

We have J' $\left(x, y \mid x_{1}, \ldots, x_{n-1}, t\right)$ is invariant under any permutation of $x_{1}, \ldots, x_{n-1}$.

For all $\mathrm{t}>0$ :

$$
\begin{aligned}
J^{\prime}\left(x, x \mid x_{1}, \ldots, x_{n-1}\right)= & \frac{\left|\left(x, x \mid x_{1}, \ldots, x_{n-1}\right)\right|}{t+\left|\left(x, x \mid x_{1}, \ldots, x_{n-1}\right)\right|} \\
& =\frac{\left|\left(x_{1} \cdot x_{1} \mid x, \ldots, x_{n-1}\right)\right|}{t+\left|\left(x_{1}, x_{1} \mid x, \ldots, x_{n-1}\right)\right|} \\
& =J^{\prime}\left(x_{1}, x_{1} \mid x, \ldots, x_{n-1}, t\right) \\
J^{\prime}\left(x, x \mid x_{1}, \ldots, x_{n-1}, \frac{t}{|a b|}\right)= & \frac{\left|\left(x, x \mid x_{1}, \ldots, x_{n-1}\right)\right|}{\frac{t}{\mid a b}|+|\left(x, x \mid x_{1}, \ldots, x_{n-1}\right)} \\
& =\frac{\left|a b \|\left(x, x \mid x_{1}, \ldots, x_{n-1}\right)\right|}{t+\left|a b \|\left(x, x \mid x_{1}, \ldots, x_{n-1}\right)\right|} \\
& =\frac{\left|\left(a x, b x \mid x_{1}, \ldots, x_{n-1}\right)\right|}{t+\left|\left(a x, b x \mid x_{1}, \ldots, x_{n-1}\right)\right|} \\
& =J^{\prime}\left(a x, b x \mid x_{1}, \ldots, x_{n-1}, t\right)
\end{aligned}
$$

If (a) $\mathrm{s}+\mathrm{t}<0$ (b) $\mathrm{s}=\mathrm{t}=0$ (c) $\mathrm{s}+\mathrm{t}>0$; $\mathrm{s}>0, \mathrm{t}<0$; $\mathrm{s}<$ $0, \mathrm{t}>0$,

Then the above relation is obvious. If (d) $s>0, t>0, s$ $+t>0$. Then Without loss of generality assume that:

$$
\begin{aligned}
& \mathrm{J}^{\prime}\left(\mathrm{x}, \mathrm{y} \mid \mathrm{x}_{1}, \ldots, \mathrm{x}_{\mathrm{n}-1}\right) \mid \leq \mathrm{J}^{\prime}\left(\mathrm{x}^{\prime}, \mathrm{y} \mid \mathrm{x}_{1}, \ldots, \mathrm{x}_{\mathrm{n}-1}\right) \\
& \frac{\left|\left(x, y \mid x_{1}, \ldots, x_{n-1}\right)\right|}{t+\left|\left(x, y \mid x_{1}, \ldots, x_{n-1}\right)\right|} \leq \frac{\left|\left(x^{\prime}, y \mid x_{1}, \ldots, x_{n-1}\right)\right|}{s+\left|\left(x^{\prime}, y \mid x_{1}, \ldots, x_{n-1}\right)\right|} \\
& \frac{t+\left|\left(x, y \mid x_{1}, \ldots, x_{n-1}\right)\right|}{\left|\left(x, y \mid x_{1}, \ldots, x_{n-1}\right)\right|} \geq \frac{s+\left|\left(x^{\prime}, y \mid x_{1}, \ldots, x_{n-1}\right)\right|}{\left|\left(x^{\prime}, y \mid x_{1}, \ldots, x_{n-1}\right)\right|} \\
& 1+\frac{t}{\left|\left(x, y \mid x_{1}, \ldots, x_{n-1}\right)\right|} \geq 1+\frac{s}{\left|\left(x^{\prime}, y \mid x_{1}, \ldots, x_{n-1}\right)\right|} \\
& \frac{\mathrm{t}}{\left|\left(\mathrm{x}, \mathrm{y} \mid \mathrm{x}_{1}, \ldots, \mathrm{x}_{\mathrm{n}-1}\right)\right|} \geq \frac{\mathrm{s}}{\left|\left(\mathrm{x}^{\prime}, \mathrm{y} \mid \mathrm{x}_{1}, \ldots, \mathrm{x}_{\mathrm{n}-1}\right)\right|} \\
& \frac{\mathrm{t}\left|\left(\mathrm{x}, \mathrm{y} \mid \mathrm{x}_{1}, \ldots, \mathrm{x}_{\mathrm{n}-1}\right)\right|}{\mathrm{s}} \geq\left|\left(\mathrm{x}, \mathrm{y} \mid \mathrm{x}_{1}, \ldots, \mathrm{x}_{\mathrm{n}-1}\right)\right|
\end{aligned}
$$

$$
\begin{aligned}
& \left|\left(x^{\prime}, y \mid x_{1}, \ldots, x_{n-1}\right)\right|+\frac{t\left|\left(x^{\prime}, y \mid x_{1}, \ldots, x_{n-1}\right)\right|}{s} \geq \\
& \left|\left(x^{\prime}, y \mid x_{1}, \ldots, x_{n-1}\right)\right|+\left|\left(x, y \mid x_{1}, \ldots, x_{n-1}\right)\right| \\
& \left(1+\frac{\mathrm{t}}{\mathrm{s}}\right)\left|\left(\mathrm{x}^{\prime}, \mathrm{y} \mid \mathrm{x}_{1}, \ldots, \mathrm{x}_{\mathrm{n}-1}\right)\right| \geq\left|\left(\mathrm{x}+\mathrm{x}^{\prime}, \mathrm{y} \mid \mathrm{x}_{1}, \ldots, \mathrm{x}_{\mathrm{n}-1}\right)\right| \\
& \left(\frac{\mathrm{s}+\mathrm{t}}{\mathrm{s}}\right)\left|\left(\mathrm{x}^{\prime}, \mathrm{y} \mid \mathrm{x}_{1}, \ldots, \mathrm{x}_{\mathrm{n}-1}\right)\right| \geq\left|\left(\mathrm{x}+\mathrm{x}^{\prime}, y \mid \mathrm{x}_{1}, \ldots, \mathrm{x}_{\mathrm{n}-1}\right)\right| \\
& \frac{\left|\left(x^{\prime}, y \mid x_{1}, \ldots, x_{n-1}\right)\right|}{s} \geq \frac{\left|\left(x+x^{\prime}, y \mid x_{1}, \ldots, x_{n-1}\right)\right|}{s+t} \\
& \frac{s}{\left|\left(x^{\prime}, y \mid x_{1}, \ldots, x_{n-1}\right)\right|} \leq \frac{s+t}{\left|\left(x+x^{\prime}, y \mid x_{1}, \ldots, x_{n-1}\right)\right|} \\
& 1+\frac{\mathrm{s}}{\left|\left(\mathrm{x}^{\prime}, \mathrm{y} \mid \mathrm{x}_{1}, \ldots, \mathrm{x}_{\mathrm{n}-1}\right)\right|} \leq 1+\frac{\mathrm{s}+\mathrm{t}}{\left|\left(\mathrm{x}+\mathrm{y} \mid \mathrm{x}_{1}, \ldots, \mathrm{x}_{\mathrm{n}-1}\right)\right|} \\
& \frac{s+\left|\left(x^{\prime}, y \mid x_{1}, \ldots, x_{n-1}\right)\right|}{\left|\left(x^{\prime}, y \mid x_{1}, \ldots, x_{n-1}\right)\right|} \leq \frac{s+t\left|\left(x+x^{\prime}, y \mid x_{1}, \ldots, x_{n-1}\right)\right|}{\left|\left(x+x^{\prime}, y \mid x_{1}, \ldots, x_{n-1}\right)\right|} \\
& \frac{\left|\left(x^{\prime}, y \mid x_{1}, \ldots, x_{n-1}\right)\right|}{s+\left|\left(x^{\prime}, y \mid x_{1}, \ldots, x_{n-1}\right)\right|} \geq \frac{\left|\left(x+x^{\prime}, y \mid x_{1}, \ldots, x_{n-1}\right)\right|}{s+t+\left|\left(x+x^{\prime}, y \mid x_{1}, \ldots, x_{n-1}\right)\right|} \\
& \max \left\{J^{\prime}\left(x, y \mid x_{1}, \ldots, x_{n-1}, t\right)\right\}, J\left(x^{\prime}, y \mid x_{1}, \ldots, x_{n-1}, s\right) \geq \\
& J^{\prime}\left(x+x^{\prime}, y \mid x_{1}, \ldots, x_{n-1}, s+t\right)
\end{aligned}
$$

Without loss of generality assume that $\mathrm{J}^{\prime}(\mathrm{x}$ $\left.\mathrm{x} \mid \mathrm{x}_{1}, \ldots, \mathrm{x}_{\mathrm{n}-1}, \mathrm{t}\right) \leq \mathrm{J}^{\prime}\left(\mathrm{y}, \mathrm{y} \mid \mathrm{x}_{1}, \ldots, \mathrm{x}_{\mathrm{n}-1}, \mathrm{~s}\right)$ for all $\mathrm{s}, \mathrm{t} \in \mathrm{R}$ with $\mathrm{s}>0, \mathrm{t}>0$ :

$$
\begin{aligned}
& \frac{\left|\left(x, x \mid x_{1}, \ldots, x_{n-1}\right)\right|}{t+\left|\left(x, x \mid x_{1}, \ldots, x_{n-1}\right)\right|} \leq \frac{\left|\left(y, y \mid x_{2}, \ldots, x_{n}\right)\right|}{s+\left|\left(y, y \mid x_{1}, \ldots, x_{n-1}\right)\right|} \\
& \frac{\mathrm{t}+\left|\left(\mathrm{x}, \mathrm{x} \mid \mathrm{x}_{1}, \ldots, \mathrm{x}_{\mathrm{n}-1}\right)\right|}{\left|\left(\mathrm{x}, \mathrm{x} \mid \mathrm{x}_{1}, \ldots, \mathrm{x}_{\mathrm{n}-1}\right)\right|} \geq \frac{\mathrm{s}+\left|\left(\mathrm{y}, \mathrm{y} \mid \mathrm{x}_{2}, \ldots, \mathrm{x}_{\mathrm{n}}\right)\right|}{\left|\left(\mathrm{y}, \mathrm{y} \mid \mathrm{x}_{1}, \ldots, \mathrm{x}_{\mathrm{n}-1}\right)\right|} \\
& 1+\frac{\mathrm{t}}{\left|\left(\mathrm{x}, \mathrm{x} \mid \mathrm{x}_{1}, \ldots, \mathrm{x}_{\mathrm{n}-1}\right)\right|} \geq 1+\frac{\mathrm{s}}{\left|\left(\mathrm{y}, \mathrm{y} \mid \mathrm{x}_{1}, \ldots, \mathrm{x}_{\mathrm{n}-1}\right)\right|} \\
& \frac{\mathrm{t}}{\left|\left(\mathrm{x}, \mathrm{x} \mid \mathrm{x}_{1}, \ldots, \mathrm{x}_{\mathrm{n}-1}\right)\right|} \geq \frac{\mathrm{s}}{\left|\left(\mathrm{y}, \mathrm{y} \mid \mathrm{x}_{1}, \ldots, \mathrm{x}_{\mathrm{n}-1}\right)\right|} \\
& \left|\left(y, y \mid x_{1}, \ldots, x_{n-1}\right)\right| \geq\left|\left(x, x \mid x_{1}, \ldots, x_{n-1}\right)\right| \cdot \frac{s}{t} \\
& \left|\left(y, y \mid x_{1}, \ldots, x_{n-1}\right)\right| \cdot\left|\left(y, y \mid x_{1}, \ldots ., x_{n-1}\right)\right| \\
& \geq\left|\left(x, x \mid x_{1}, \ldots, x_{n-1}\right)\right|\left|\left(y, y \mid x_{1}, \ldots, x_{n-1}\right)\right| \cdot \frac{s}{t}
\end{aligned}
$$

\section{By Remark:}

$$
\begin{aligned}
& \frac{s\left|\left(\mathrm{x}, \mathrm{y} \mid \mathrm{x}_{1}, \ldots, \mathrm{x}_{\mathrm{n}-1}\right)\right|^{2}}{\mathrm{t}} \leq\left|\left(\mathrm{y}, \mathrm{y} \mid \mathrm{x}_{1}, \ldots, \mathrm{x}_{\mathrm{n}-1}\right)\right|^{2} \\
& \frac{\left|\left(\mathrm{x}, \mathrm{y} \mid \mathrm{x}_{1}, \ldots, \mathrm{x}_{\mathrm{n}-1}\right)\right|^{2} \cdot s}{\mathrm{st}} \leq \frac{\left|\left(\mathrm{y}, \mathrm{y} \mid \mathrm{x}_{1}, \ldots, \mathrm{x}_{\mathrm{n}-1}\right)\right|^{2}}{\mathrm{~s}}
\end{aligned}
$$




$$
\frac{\left|\left(\mathrm{x}, \mathrm{y} \mid \mathrm{x}_{1}, \ldots, \mathrm{x}_{\mathrm{n}-1}\right)\right|^{2}}{\mathrm{st}} \leq \frac{\left|\left(\mathrm{y}, \mathrm{y} \mid \mathrm{x}_{1}, \ldots, \mathrm{x}_{\mathrm{n}-1}\right)\right|^{2}}{\mathrm{~s}^{2}}
$$

Taking square root on both sides:

$$
\begin{aligned}
& \frac{\left|\left(\mathrm{x}, \mathrm{x} \mid \mathrm{x}_{1}, \ldots, \mathrm{x}_{\mathrm{n}-1}\right)\right|}{\sqrt{\mathrm{st}}} \leq \frac{\left|\left(\mathrm{y}, \mathrm{y} \mid \mathrm{x}_{1}, \ldots, \mathrm{x}_{\mathrm{n}-1}\right)\right|}{\mathrm{s}} \\
& \frac{\mathrm{s}}{\left|\left(\mathrm{y}, \mathrm{y} \mid \mathrm{x}_{1}, \ldots, \mathrm{x}_{\mathrm{n}-1}\right)\right|} \leq \frac{\sqrt{\mathrm{st}}}{\left|\left(\mathrm{x}, \mathrm{y} \mid \mathrm{x}_{1}, \ldots, \mathrm{x}_{\mathrm{n}-1}\right)\right|} \\
& 1+\frac{\mathrm{s}}{\left|\left(\mathrm{y}, \mathrm{y} \mid \mathrm{x}_{1}, \ldots, \mathrm{x}_{\mathrm{n}-1}\right)\right|} \leq 1+\frac{\sqrt{\mathrm{st}}}{\left|\left(\mathrm{x}, \mathrm{y} \mid \mathrm{x}_{1}, \ldots, \mathrm{x}_{\mathrm{n}-1}\right)\right|} \\
& \frac{\left|\left(y, y \mid x_{1}, \ldots, x_{n-1}\right)\right|+s}{\left|\left(y, y \mid x ~ 1, \ldots, x_{n-1}\right)\right|} \leq \frac{\left|\left(x, y \mid x_{1}, \ldots, x_{n-1}\right)\right|+\sqrt{s t}}{\left|\left(x, y \mid x_{1}, \ldots, x_{n-1}\right)\right|} \\
& \frac{\left|\left(y, y \mid x_{1}, \ldots, x_{n-1}\right)\right|}{s+\left|\left(y, y \mid x_{1}, \ldots, x_{n-1}\right)\right|} \geq \frac{\left|\left(x, y \mid x_{1}, \ldots, x_{n-1}\right)\right|}{\sqrt{s t}+\left|\left(x, y \mid x_{1}, \ldots, x_{n-1}\right)\right|} \\
& \max \left\{\mathrm{J}^{\prime}\left(\mathrm{x}, \mathrm{x}, \mid \mathrm{x} 1, \ldots, \mathrm{x}_{\mathrm{n}-1}\right), \mathrm{J}^{\prime}\left(\mathrm{y}, \mathrm{y} \mid \mathrm{x} 1, \ldots, \mathrm{x}_{\mathrm{n}-1}\right)\right\} \\
& \geq J^{\prime}\left(x, y \mid x_{1} x_{n-1}, \sqrt{t s}\right)
\end{aligned}
$$

For all $\mathrm{t} 1<\mathrm{t}_{2} \leq 0$ then, by our definition:

$$
J^{\prime}\left(x, y \mid x_{1}, \ldots, x_{n-1}, t_{1}\right)=J^{\prime}\left(x, y \mid x_{1}, \ldots, x_{n-1}, t_{2}\right)=1
$$

Suppose $t_{2}>t_{1}>0$ then:

$$
\begin{aligned}
& \frac{\left|\left(x, y \mid x_{1}, \ldots, x_{n-1}\right)\right|}{t_{1}+\left|\left(x, y \mid x_{1}, \ldots, x_{n-1}\right)\right|}-\frac{\left|\left(x, y \mid x_{1}, \ldots, x_{n-1}\right)\right|}{t_{2}+\left|\left(x, y \mid x_{1}, \ldots, x_{n-1}\right)\right|} \\
& =\frac{\left|\left(x, y \mid x_{1}, \ldots, x_{n-1}\right)\right|\left(t_{2}-t_{1}\right)}{\left(t_{2}+\left|\left(x, y \mid x_{1}, \ldots, x_{n-1}\right)\right|\right)\left(t_{1}+\left|\left(x, y \mid x_{1}, \ldots, x_{n-1}\right)\right|\right)} \\
& \geq 0, \text { for all }\left(x, y \mid x_{1}, \ldots, x_{n-1}\right) \in X^{n+1} \\
& \frac{\left|\left(x, y \mid x_{1}, \ldots, x_{n-1}\right)\right|}{t+\left|\left(x, y \mid x_{1}, \ldots, x_{n-1}\right)\right|} \geq \frac{\left|\left(x, y \mid x_{1}, \ldots, x_{n-1}\right)\right|}{t_{2}+\left|\left(x, y \mid x_{1}, \ldots, x_{n-1}\right)\right|} \\
& J^{\prime}\left(x, y \mid x_{1}, \ldots, x_{n-1}, t_{1}\right) \geq J^{\prime}\left(x, y \mid x_{1}, \ldots, x_{n-1}, t_{2}\right)
\end{aligned}
$$

Thus $\mathrm{J}^{\prime}\left(\mathrm{x} \mathrm{y} \mid \mathrm{x}_{1}, \ldots, \mathrm{x}_{\mathrm{n}-1}, \mathrm{t}\right)$ is non-increasing function. Also:

$$
\begin{aligned}
& \lim _{t \rightarrow \infty} J^{\prime}\left(x, y \mid x_{1}, \ldots, x_{n-1}, t\right)=\lim _{t \rightarrow \infty} \frac{\left|\left(x, y \mid x_{1}, \ldots, x_{n-1}\right)\right|}{t+\left|\left(x, y \mid x_{1}, \ldots, x_{n-1}\right)\right|} \\
& \lim _{t \rightarrow \infty} J^{\prime} \frac{\left|\left(x, y \mid x_{1}, \ldots, x_{n-1}\right)\right|}{t\left(1+\frac{1}{t}\left|\left(x, y \mid x_{2}, \ldots, x_{n-1}\right)\right|\right)}=0 .
\end{aligned}
$$

Definition 13: We call that t-norm $\Delta$ and t-conorm $\nabla$ are h-type if the family of functions $\left\{\Delta^{\mathrm{m}}(\mathrm{t})\right\}_{\mathrm{m}=1}^{\infty}$ and $\left\{\nabla^{\mathrm{m}}(\mathrm{t})\right\}_{\mathrm{m}=1}^{\infty}$ are equi-continuous at $\mathrm{t}=1$ and $\mathrm{t}=0$ respectively.

Where:

$$
\begin{aligned}
& \Delta^{1}(\mathrm{t})=\Delta(\mathrm{t}, \mathrm{t}), \Delta^{\mathrm{m}}(\mathrm{t})=\Delta\left(\mathrm{t}, \Delta^{\mathrm{m}-1}(\mathrm{t})\right) \text { and } \\
& \nabla^{1}(\mathrm{t})=\nabla(\mathrm{t}, \mathrm{t}), \nabla^{\mathrm{m}}(\mathrm{t})=\nabla\left(\mathrm{t}, \nabla^{\mathrm{m}-1}(\mathrm{t})\right) \\
& \mathrm{t} \in[0,1], \mathrm{m}=2,3, \ldots
\end{aligned}
$$

Definition 14: Let $(X, J, J ', \Delta, \nabla)$ be a IF-n-IP-space. (i) A sequence $x_{n} \subset X$ is said to converge to $x \in X$ if $\forall$ $\varepsilon>0, \forall a \in(0,1], \Xi N$, when

$\mathrm{n} \geq \mathrm{N}, \mathrm{J}\left(\mathrm{x}_{\mathrm{n}}-\mathrm{x}, \mathrm{x}_{\mathrm{n}}-\mathrm{x} \mid \mathrm{x}_{1}, \ldots, \mathrm{x}_{\mathrm{n}-1} \varepsilon\right)>1-\mathrm{a}$ and J' $\left(\mathrm{x}_{\mathrm{n}}-\mathrm{x}, \mathrm{x}_{\mathrm{n}^{-}}\right.$ $\left.\mathrm{x} \mid \mathrm{x}_{1}, \ldots, \mathrm{x}_{\mathrm{n}-1} \varepsilon\right) \leq \mathrm{a}$.

(ii) A sequence $x_{n} \subset X$ is called a Cauchy sequence if $\forall \varepsilon>0, \forall \mathrm{a} \in(0,1], \Xi \mathrm{N}$, when $\mathrm{m}, \mathrm{n} \geq \mathrm{N}, \mathrm{J}\left(\mathrm{x}_{\mathrm{n}}-\mathrm{x}_{\mathrm{m}}, \mathrm{x}_{\mathrm{n}}-\mathrm{x}_{\mathrm{m}} \mid \mathrm{x}_{1}, \ldots, \mathrm{x}_{\mathrm{n}-1} \varepsilon\right)>1-\mathrm{a}$ and:

$\mathrm{J}^{\prime}\left(\mathrm{x}_{\mathrm{n}}-\mathrm{x}_{\mathrm{m}}, \mathrm{x}_{\mathrm{n}}-\mathrm{x}_{\mathrm{m}} \mid \mathrm{x}_{1}, \ldots, \mathrm{x}_{\mathrm{n}-1}, \varepsilon\right) \leq \mathrm{a}$

\section{CONCLUSION}

Theorem 15: Let (X, J, J', $\Delta, \nabla)$ be a complete IF-n-IPspace and $\Delta, \nabla$ be t-norm and t-conorm of h-type respectively. Let $\mathrm{T}:(\mathrm{X}, \mathrm{J}, \mathrm{J}, \Delta, \nabla) \rightarrow\left(\mathrm{X}, \mathrm{J}, \mathrm{J}^{\prime}, \Delta, \nabla\right)$ be a linear mapping satisfying the following condition:

$$
J\left(T x, y \mid x_{1}, \ldots, x_{n-1}, t\right) \geq J\left(x, y\left|x_{1}, \ldots, x_{n-1}\right|, \frac{t}{k(\alpha, \beta)}\right)
$$

And:

$$
\mathrm{J}\left(\mathrm{Tx}, \mathrm{y} \mid \mathrm{x}_{1}, \ldots, \mathrm{x}_{\mathrm{n}-1}, \mathrm{t}\right) \leq \mathrm{J}^{\prime}\left(\mathrm{x}, \mathrm{y} \mid \mathrm{x}_{1}, \ldots, \mathrm{x}_{\mathrm{n}-1}, \frac{\mathrm{t}}{\mathrm{k}(\alpha, \beta)}\right)
$$

For all $\mathrm{x}, \mathrm{y}, \mathrm{x}_{1}, \mathrm{x}_{2}, \ldots, \mathrm{x}_{\mathrm{n}-1} \in \mathrm{X} \mathrm{t} \geq 0 \alpha, \beta \in(0, \infty)$ and $\mathrm{k}$ $(\alpha, \beta):(0,+\infty) \times(0,+\infty) \rightarrow(0,1)$ is a function. Then $\mathrm{T}$ has exactly one fixed point $\mathrm{X}_{*} \in \mathrm{X}$. Furthermore, for any $x_{0} \in X$, the iterative sequence $\left\{T^{n} x_{0}\right\} \tau$-converges to $\mathrm{X} *$.

Proof: Firstly, we prove that for any $x_{0} \in X$, the sequence $\left\{x_{m}\right\}_{m=0}^{\infty}$ is at $\tau$ Cauchy sequence, where $\left\{\mathrm{x}_{\mathrm{m}}\right\}_{\mathrm{m}=0}^{\infty}=\left\{\mathrm{x}_{0}, \mathrm{x}_{1}=\mathrm{Tx}_{0}, \ldots, \mathrm{x}_{\mathrm{m}}=\mathrm{T}^{\mathrm{m}} \mathrm{x}_{0}, ..\right\}$

By (8) and (17) of Definition 11, we have:

$$
\begin{aligned}
& J\left(x_{0}-T^{m} x_{0}, y \mid x_{1}, \ldots, x_{n-1}, \frac{t}{k(\alpha, \beta)}\right)= \\
& J\left(x_{0}-T_{0}+T x_{0}-T^{m} x_{0}, y \mid x_{1}, \ldots, x_{n-1}, \frac{t}{k(\alpha, \beta)}\right)
\end{aligned}
$$


J. Math. \& Stat., 8 (2): 198-210, 2012

$$
\begin{aligned}
& \geq \Delta\left(\mathrm{J}\left(\mathrm{x}_{0}-\mathrm{Tx}_{0}, \mathrm{y} \mid \mathrm{x}_{1}, \ldots, \mathrm{x}_{\mathrm{n}-1}, \frac{\mathrm{t}(1-\mathrm{k}(\mathrm{a}, \beta))}{\mathrm{k}(\mathrm{a}, \beta)}\right)\right) \\
& \left.J\left(\mathrm{Tx}_{0}-\mathrm{T}^{\mathrm{m}} \mathrm{x}_{0}, \mathrm{y} \mid \mathrm{x}_{1}, \ldots, \mathrm{x}_{\mathrm{n}-1}, \frac{\mathrm{tk}(\mathrm{a}, \beta)}{\mathrm{k}(\mathrm{a}, \beta)}\right)\right) \\
& \geq \Delta\left(\mathrm{J}\left(\mathrm{x}_{0}-\mathrm{Tx}_{0}, \mathrm{y} \mid \mathrm{x}_{1}, \ldots, \mathrm{x}_{\mathrm{n}-1}, \frac{\mathrm{t}(1-\mathrm{k}(\mathrm{a}, \beta))}{\mathrm{k}(\mathrm{a}, \beta)}\right)\right. \\
& J\left(x_{0}-T^{m-1} x_{0}, y \mid x_{1}, \ldots, x_{n-1}, \frac{t}{k(a, \phi)}\right) \\
& =\Delta\left(\mathrm{J}\left(\mathrm{x}_{0}-\mathrm{Tx}_{0}, \mathrm{y} \mid \mathrm{x}_{1}, \ldots, \mathrm{x}_{\mathrm{n}-1}, \frac{\mathrm{t}(1-\mathrm{k}(\mathrm{a}, \beta))}{\mathrm{k}(\mathrm{a}, \beta)}\right)\right. \\
& \left.J\left(x_{0}-T_{0}+T_{0}-T^{m-1} x_{0}, y \mid x_{1}, \ldots, x_{n-1}, \frac{t}{k(a, \beta)}\right)\right) \\
& \geq \Delta\left(\mathrm{J}\left(\mathrm{x}_{0}-\mathrm{Tx}_{0}, \mathrm{y} \mid \mathrm{x}_{1}, \ldots, \mathrm{x}_{\mathrm{n}-1}, \frac{\mathrm{t}(1-\mathrm{k}(\mathrm{a}, \beta))}{\mathrm{k}(\mathrm{a}, \beta)}\right),\right. \\
& \Delta\left(\mathrm{J}\left(\mathrm{x}_{0}-\mathrm{Tx}_{0}, \mathrm{y} \mid \mathrm{x}_{1}, \ldots, \mathrm{x}_{\mathrm{n}-1}, \frac{\mathrm{t}(1-\mathrm{k}(\mathrm{a}, \beta))}{\mathrm{k}(\mathrm{a}, \beta)}\right)\right. \\
& \left.\left.J\left(\operatorname{Tx}_{0}-\mathrm{T}^{\mathrm{m}-1} \mathrm{x}_{0}, \mathrm{y} \mid \mathrm{x}_{1}, \ldots, \mathrm{x}_{\mathrm{n}-1}, \frac{\mathrm{tk}(\mathrm{a}, \beta)}{\mathrm{k}(\mathrm{a}, \boldsymbol{\beta})}\right)\right)\right) \\
& \geq \Delta\left(\mathrm{J}\left(\mathrm{x}_{0}-\mathrm{Tx}_{0}, \mathrm{y} \mid \mathrm{x}_{1}, \ldots, \mathrm{x}_{\mathrm{n}-1}, \frac{\mathrm{t}(1-\mathrm{k}(\mathrm{a}, \beta))}{\mathrm{k}(\mathrm{a}, \beta)}\right)\right. \\
& , \Delta\left(\mathrm{J}\left(\mathrm{x}_{0}-\mathrm{Tx}_{0}, \mathrm{y} \mid \mathrm{x}_{1}, \ldots, \mathrm{x}_{\mathrm{n}-1}, \frac{\mathrm{t}(1-\mathrm{k}(\mathrm{a}, \beta))}{\mathrm{k}(\mathrm{a}, \beta)}\right)\right. \\
& \left.\left.J,\left(x_{0}-T^{m-2} x_{0}, y \mid x_{1}, \ldots, x_{n-1}, \frac{t}{k(a, \beta)}\right)\right)\right) \\
& \geq \ldots . \geq \Delta\left(\mathrm{J}\left(\mathrm{x}_{0}-\mathrm{Tx}_{0}, \mathrm{y} \mid \mathrm{x}_{1}, \ldots, \mathrm{x}_{\mathrm{n}}, \frac{\mathrm{t}(1-\mathrm{k}(\mathrm{a}, \beta))}{\mathrm{k}(\mathrm{a}, \beta)}\right)\right. \\
& , \Delta\left(\mathrm{J}\left(\mathrm{x}_{0}-\mathrm{Tx}_{0}, \mathrm{y} \mid \mathrm{x}_{1}, \ldots, \mathrm{x}_{\mathrm{n}-1}, \frac{\mathrm{t}(1-\mathrm{k}(\mathrm{a}, \beta))}{\mathrm{k}(\mathrm{a}, \phi)}\right)\right.
\end{aligned}
$$

And:

$$
\begin{aligned}
& J^{\prime}\left(x_{0}-T^{m} x_{0}, y \mid x_{1}, \ldots, x_{n-1}, \frac{t}{k(\alpha, \beta)}\right)= \\
& J^{\prime}\left(x_{0}-T x_{0}+T x_{0}-T^{m} x_{0}, y \mid x_{1}, \ldots, x_{n-1}, \frac{t}{k(\alpha, \beta)}\right) \\
& \quad \leq\left(J^{\prime}\left(x_{0}-T x_{0}, y \mid x_{1}, \ldots, x_{n-1}, \frac{t(1-k(a, \beta))}{k(a, \beta)}\right)\right. \\
& \left.\quad J^{\prime}\left(T x_{0}-T^{m} x_{0}, y \mid x_{1}, \ldots, x_{n-1}, \frac{t k(a, \beta)}{k(a, \beta)}\right)\right) \\
& \leq \nabla\left(J^{\prime}\left(x_{0}-T x_{0}, y \mid x_{1}, \ldots, x_{n-1}, \frac{t(1-k(\alpha, \beta))}{k(\alpha, \beta)}\right)\right) \\
& \left.J^{\prime}\left(x_{0}-T^{m-1} x_{0}, y \mid x_{1}, \ldots, x_{n-1} \frac{t}{k(\alpha, \beta)}\right)\right)
\end{aligned}
$$

$$
\begin{aligned}
& =\nabla\left(\mathrm{J}^{\prime}\left(\mathrm{x}_{0}-\mathrm{Tx}_{0}, \mathrm{y} \mid \mathrm{x}_{1}, \ldots, \mathrm{x}_{\mathrm{n}-1}, \frac{\mathrm{t}(1-\mathrm{k}(\alpha, \beta))}{\mathrm{k}(\alpha, \beta)}\right)\right) \\
& \left.J^{\prime}\left(x_{0}-\mathrm{Tx}_{0}+\mathrm{T}-\mathrm{T}^{\mathrm{m}-1} \mathrm{x}_{0}, \mathrm{y} \mid \mathrm{x}_{1}, \ldots, \mathrm{x}_{\mathrm{n}-1} \frac{\mathrm{t}}{\mathrm{k}(\alpha, \beta)}\right)\right) \\
& \leq \nabla\left(\mathrm{J}^{\prime}\left(\mathrm{x}_{0}-\mathrm{Tx}_{0}, \mathrm{y} \mid \mathrm{x}_{1}, \ldots, \mathrm{x}_{\mathrm{n}-1}, \frac{\mathrm{t}(1-\mathrm{k}(\mathrm{a}, \beta))}{\mathrm{k}(\mathrm{a}, \beta)}\right)\right. \\
& , \nabla\left(\mathrm{J}^{\prime}\left(\mathrm{x}_{0}-\mathrm{Tx}_{0}, \mathrm{y} \mid \mathrm{x}_{1}, \ldots, \mathrm{x}_{\mathrm{n}-1}, \frac{\mathrm{t}(1-\mathrm{k}(\mathrm{a}, \boldsymbol{\beta}))}{\mathrm{k}(\mathrm{a}, \boldsymbol{\beta})}\right)\right. \\
& \left.\left.J^{\prime}\left(\operatorname{Tx}_{0}-\mathrm{T}^{\mathrm{m}-1} \mathrm{x}_{0}, \mathrm{y} \mid \mathrm{x}_{1}, \ldots, \mathrm{x}_{\mathrm{n}-1}, \frac{\mathrm{tk}(\mathrm{a}, \beta)}{\mathrm{k}(\mathrm{a}, \beta)}\right)\right)\right) \\
& \leq \nabla\left(\mathrm{J}^{\prime}\left(\mathrm{x}_{0}-\mathrm{Tx}_{0}, \mathrm{y} \mid \mathrm{x}_{1}, \ldots, \mathrm{x}_{\mathrm{n}-1}, \frac{\mathrm{t}(1-\mathrm{k}(\mathrm{a}, \beta))}{\mathrm{k}(\mathrm{a}, \beta)}\right)\right. \\
& , \nabla\left(J^{\prime}\left(x_{0}-\mathrm{Tx}_{0}, \mathrm{y} \mid \mathrm{x}_{1}, \ldots, \mathrm{x}_{\mathrm{n}-1}, \frac{\mathrm{t}(1-\mathrm{k}(\mathrm{a}, \beta))}{\mathrm{k}(\mathrm{a}, \beta)}\right),\right. \\
& J^{\prime}\left(x_{0}-T^{m-2} x_{0}, y \mid x_{1}, \ldots, x_{n-1}, \frac{t}{k(a, \beta)}\right) \\
& \leq \ldots \leq \nabla\left(\mathrm{J}^{\prime}\left(\mathrm{x}_{0}-\mathrm{Tx}_{0}, \mathrm{y} \mid \mathrm{x}_{1}, \ldots, \mathrm{x}_{\mathrm{n}-1}, \frac{\mathrm{t}(1-\mathrm{k}(\mathrm{a}, \beta))}{\mathrm{k}(\mathrm{a}, \beta)}\right),\right. \\
& \nabla\left(J^{\prime}\left(x_{0}-\mathrm{Tx}_{0}, \mathrm{y} \mid \mathrm{x}_{1}, \ldots, \mathrm{x}_{\mathrm{n}-1}, \frac{\mathrm{t}(1-\mathrm{k}(\mathrm{a}, \beta))}{\mathrm{k}(\mathrm{a}, \boldsymbol{\beta})}\right)\right. \\
& \nabla\left(\ldots, \nabla\left(\mathrm{J}^{\prime}\left(\mathrm{x}_{0}-\mathrm{Tx}_{0}, \mathrm{y} \mid \mathrm{x}_{1}, \ldots, \mathrm{x}_{\mathrm{n}-1}, \frac{\mathrm{t}(1-\mathrm{k}(\mathrm{a}, \beta))}{\mathrm{k}(\mathrm{a}, \boldsymbol{\beta})}\right)\right.\right. \\
& , J^{\prime}\left(x_{0}-T_{0}, y \mid x_{1}, \ldots, x_{n-1}, \frac{t}{k(a, \beta)}\right)
\end{aligned}
$$

Because of $\mathrm{k}(\alpha, \beta) \in(0,1)$, therefore we get:

$$
\frac{\mathrm{t}(1-\mathrm{k}(\alpha, \beta))}{\mathrm{k}(\alpha, \beta)} \leq \frac{\mathrm{t}}{\mathrm{k}(\alpha, \beta)}
$$

As J, J' are non-decreasing and non-increasing respectively, Therefore, we have:

By the property of t-norm and t-conorm, we obtain:

$$
\begin{gathered}
\mathrm{J}\left(\mathrm{x}_{0}-\mathrm{T}^{\mathrm{m}} \mathrm{x}_{0}, \mathrm{y} \mid \mathrm{x}_{1}, \ldots, \mathrm{x}_{\mathrm{n}-1}, \frac{\mathrm{t}}{\mathrm{k}(\alpha, \beta)}\right) \\
\geq \Delta\left(\mathrm{J}\left(\mathrm{x}_{0}-\mathrm{T} \mathrm{x}_{0}, \mathrm{y} \mid \mathrm{x}_{1}, \ldots, \mathrm{x}_{\mathrm{n}-1} \frac{\mathrm{t}(1-\mathrm{k}(\alpha, \beta))}{\mathrm{k}(\alpha, \beta)}\right)\right. \\
, \Delta\left(\mathrm{J}\left(\mathrm{x}_{0}-\mathrm{Tx}_{0}, \mathrm{y} \mid \mathrm{x}_{1}, \ldots, \mathrm{x}_{\mathrm{n}-1}, \frac{\mathrm{t}(1-\mathrm{k}(\alpha, \beta))}{\mathrm{k}(\alpha, \beta)}\right)\right. \\
\Delta\left(\ldots, \Delta\left(\mathrm{J}\left(\mathrm{x}_{0}-\mathrm{Tx}_{0}, \mathrm{y} \mid \mathrm{x}_{1}, \ldots, \mathrm{x}_{\mathrm{n}-1}, \frac{\mathrm{t}(1-\mathrm{k}(\alpha, \beta))}{\mathrm{k}(\alpha, \beta)}\right)\right.\right. \\
\left.\left., J\left(\mathrm{x}_{0}-\mathrm{Tx}_{0}, \mathrm{y} \mid \mathrm{x}_{1}, \ldots, \mathrm{x}_{\mathrm{n}-1}, \frac{\mathrm{t}}{\mathrm{k}(\alpha, \beta)}\right)\right) \ldots . .\right)
\end{gathered}
$$




$$
\begin{aligned}
& =\Delta^{\mathrm{m}-1}\left(\mathrm{~J}\left(\mathrm{x}_{0}-\mathrm{Tx}_{0}, \mathrm{y} \mid \mathrm{x}_{1}, \ldots, \mathrm{x}_{\mathrm{n}-1}, \frac{\mathrm{t}(1-\mathrm{k}(\alpha, \beta))}{\mathrm{k}(\alpha, \beta)}\right)\right) \\
& J^{\prime}\left(x_{0}-T^{m} x_{0}, y \mid x_{1}, \ldots, x_{n-1}, \frac{t}{k(\alpha, \beta)}\right) \\
& \leq\left(\mathrm{J}^{\prime}\left(\mathrm{x}_{0}-\mathrm{Tx}_{0}, \mathrm{y} \mid \mathrm{x}_{1}, \ldots, \mathrm{x}_{\mathrm{n}-1}, \frac{\mathrm{t}(1-\mathrm{k}(\alpha, \beta))}{\mathrm{k}(\alpha, \beta)}\right)\right. \\
& , \nabla\left(\mathrm{J}^{\prime}\left(\mathrm{x}_{0}-\mathrm{Tx}_{0}, \mathrm{y} \mid \mathrm{x}_{1}, \ldots, \mathrm{x}_{\mathrm{n}-1}, \frac{\mathrm{t}(1-\mathrm{k}(\alpha, \beta))}{\mathrm{k}(\alpha, \beta)}\right)\right. \\
& \nabla\left(\ldots, \nabla\left(\mathrm{J}^{\prime}\left(\mathrm{x}_{0}-\mathrm{Tx}_{0}, \mathrm{y} \mid \mathrm{x}_{1}, \ldots, \mathrm{x}_{\mathrm{n}-1}, \frac{\mathrm{t}(1-\mathrm{k}(\alpha, \beta))}{\mathrm{k}(\alpha, \beta)}\right)\right), \mathrm{J}^{\prime}\right. \\
& \left(\mathrm{x}_{0},-\mathrm{Tx}_{0}, \mathrm{y} \mid \mathrm{x}_{1}, \ldots, \mathrm{x}_{\mathrm{n}-1}, \frac{\mathrm{t}(1-\mathrm{k}(\alpha, \beta))}{\mathrm{k}(\alpha, \beta)}\right) \\
& =\nabla^{\mathrm{m}-1}\left(\mathrm{~J}^{\prime}\left(\mathrm{x}_{0}-\mathrm{Tx}_{0}, \mathrm{y} \mid \mathrm{x}_{1}, \ldots, \mathrm{x}_{\mathrm{n}-1}, \frac{\mathrm{t}(1-\mathrm{k}(\alpha, \beta))}{\mathrm{k}(\alpha, \beta)}\right)\right)
\end{aligned}
$$

So, for any positive integer $\mathrm{m}, \mathrm{n}$, we have:

$$
\begin{aligned}
& J\left(T^{n} x_{0}-T^{m+n} x_{0}, T^{n} x_{0}-T^{m+n} x_{0} \mid x_{1}, . ., x_{n-1}, \frac{t}{k(\alpha, \beta)}\right) \geq \\
& J\left(x_{0}-T^{m} x_{0}-T^{m+n} x_{0} \mid x_{1}, \ldots, x_{n-1}, \frac{t}{k^{n+1}(\alpha, \beta)}\right) \\
& \geq J\left(x_{0}-T^{m} x_{0}, x_{0} T^{m} x_{0} \mid x_{1}, \ldots, x_{n-1}, \frac{t}{k^{2 n+1}(\alpha, \beta)}\right) \\
& \geq \Delta^{\mathrm{m}-1}\left(\mathrm{~J}\left(\mathrm{x}_{0}-\mathrm{Tx}_{0}, \mathrm{x}_{0}-\mathrm{T}^{\mathrm{m}} \mathrm{x}_{0} \mid \mathrm{x}_{1}, \ldots, \mathrm{x}_{\mathrm{n}-1}, \frac{\mathrm{t}(1-\mathrm{k}(\alpha, \beta))}{\mathrm{k}^{2 \mathrm{n}-1}(\alpha, \beta)}\right)\right) \\
& \geq \Delta^{\mathrm{m}-1}\left(\Delta^{\mathrm{m}-1}\left(\mathrm{~J}\left(\mathrm{x}_{0}-\mathrm{Tx}_{0}, \mathrm{x}_{0}-\mathrm{Tx}_{0} \mid \mathrm{x}_{1}, \ldots, \mathrm{x}_{\mathrm{n}-1}, \frac{\mathrm{t}(1-\mathrm{k}(\alpha, \beta))}{\mathrm{k}^{2 \mathrm{n}-1}(\alpha, \beta)}\right)\right)\right) \\
& =\Delta^{2 \mathrm{~m}-2}\left(\mathrm{~J}\left(\mathrm{x}_{0}-\mathrm{Tx}_{0}, \mathrm{x}_{0}-\mathrm{Tx}_{0} \mid \mathrm{x}_{1}, \ldots, \mathrm{x}_{\mathrm{n}-1} \frac{\mathrm{t}(1-\mathrm{k}(\alpha, \beta))^{2}}{\mathrm{k} 2^{\mathrm{n}-1}(\alpha, \beta)}\right)\right) \text {, } \\
& J^{\prime}\left(T^{n} x_{0}-T^{m+n} x_{0}, T^{n} x_{0}-T^{m+n} x_{0} \mid x_{1}, \ldots, x_{n-1}, \frac{t}{k(\alpha, \beta)}\right) \\
& \leq \mathrm{J}^{\prime}\left(\mathrm{x}_{0}-\mathrm{T}^{\mathrm{m}} \mathrm{x}_{0}, \mathrm{~T}^{\mathrm{n}} \mathrm{x}_{0}-\mathrm{T}^{\mathrm{m}+\mathrm{n}} \mathrm{x}_{0} \mid \mathrm{x}_{1}, \ldots, \mathrm{x}_{\mathrm{n}-1}, \frac{\mathrm{t}}{\mathrm{k}^{\mathrm{n}+1}(\alpha, \beta)}\right) \\
& \leq \mathrm{J}^{\prime}\left(\mathrm{x}_{0}-\mathrm{T}^{\mathrm{m}} \mathrm{x}_{0}, \mathrm{x}_{0}-\mathrm{T}^{\mathrm{m}} \mathrm{x}_{0} \mid \mathrm{x}_{1}, \ldots, \mathrm{x}_{\mathrm{n}-1}, \frac{\mathrm{t}}{\mathrm{k}^{2 \mathrm{n}-1}(\alpha, \beta)}\right) \\
& \leq \nabla^{\mathrm{m}-1}\left(\mathrm{~J}\left(\mathrm{x}_{0}-\mathrm{Tx}_{0}, \mathrm{x}_{0}-\mathrm{T}^{\mathrm{m}} \mathrm{x}_{0} \mid \mathrm{x}_{1}, \ldots, \mathrm{x}_{\mathrm{n}-1}, \frac{\mathrm{t}(1-\mathrm{k}(\alpha, \beta))}{\mathrm{k}^{2 \mathrm{n}+1}(\alpha, \beta)}\right)\right) \\
& \leq \nabla^{\mathrm{m}-1}\left(\nabla^{\mathrm{m}-1}\left(\mathrm{~J}\left(\mathrm{x}_{0}-\mathrm{Tx}_{0}, \mathrm{x}_{0}-\mathrm{Tx}_{0} \mid \mathrm{x}_{1}, \ldots, \mathrm{x}_{\mathrm{n}-1}, \frac{\mathrm{t}(1-\mathrm{k}(\alpha, \beta))^{2}}{\mathrm{~K}^{2 \mathrm{n}+1}(\alpha, \beta)}\right)\right)\right) \\
& =\nabla^{2 \mathrm{~m}-1}\left(\mathrm{~J}\left(\mathrm{x}_{0}-\mathrm{Tx}_{0}, \mathrm{x}_{0}-\mathrm{Tx}_{0} \mid \mathrm{x}_{1}, \ldots, \mathrm{x}_{\mathrm{n}-1}, \frac{\mathrm{t}(1-\mathrm{k}(\alpha, \beta))^{2}}{\mathrm{k}^{2 \mathrm{n}+1}(\alpha, \beta)}\right)\right)
\end{aligned}
$$

Note that $\Delta, \nabla$ are t-norm and t-conorm of h-type, the family of functions $\left\{\Delta^{\mathrm{m}}(\mathrm{p})\right\}_{\mathrm{m}=1}^{\infty}$ and $\left\{\Delta^{\mathrm{m}}(\mathrm{p})\right\}_{\mathrm{m}=1}^{\infty}$ is equi-continuous at $\mathrm{p}=1$ and $\mathrm{p}=0$ respt. and the functions J, J' are non-decreasing, non-increasing with:

$$
\begin{aligned}
& \lim _{t \rightarrow \infty} J\left(x, y \mid x_{1}, \ldots, x_{n-1}, t\right)= \\
& 1 \text { and } \lim _{t \rightarrow \infty} J^{\prime}\left(x, y \mid x_{1}, \ldots, x_{n-1}, t\right)=0,
\end{aligned}
$$

Then we have:

$$
\begin{aligned}
& \lim _{n \rightarrow \infty} J\left(T^{n} x_{0}-T^{m+n} x_{0}, T^{n} x_{0}-T^{m+n} x_{0} \mid x_{1}, \ldots, x_{n-1}, \frac{t}{k(\alpha, \beta)}\right) \\
& \geq \lim _{n \rightarrow \infty} \Delta^{2 m-2}\left(J\left(x_{0}-T x_{0}, x_{0}-T x_{0} \mid x_{1}, \ldots, x_{n-1}, \frac{t(1-k(\alpha, \beta))}{k^{2 n+1}(\alpha, \beta)}\right)\right) \\
& \lim _{n \rightarrow \infty} J^{\prime}\left(T^{n} x_{0}-T^{m+n} x_{0}, T^{n} x_{0}-T^{m+n} x_{0} \mid x_{1}, \ldots, x_{n-1}, \frac{t}{k(\alpha, \beta)}\right) \\
& \leq \lim _{n \rightarrow \infty} \nabla^{2 m-2}\left(J^{\prime}\left(x_{0}-T x_{0}, x_{0}-T x_{0} \mid x_{1}, \ldots, x_{n-1}, \frac{t(1-k(\alpha, \beta))}{k^{2 n+1}(\alpha, \beta)}\right)\right)
\end{aligned}
$$

By $((2),(3))$ and $((11,12))$ of Definition 11, we have $\left\{\mathrm{T}^{\mathrm{m}} \mathrm{x}_{0}\right\}_{\mathrm{m}=1}^{\infty}$ is a Cauchy sequence in $\mathrm{X}$. By the completeness of $\mathrm{X}$, let $\mathrm{x}_{\mathrm{m}} \rightarrow \mathrm{X} * \mathrm{X}(\mathrm{m} \rightarrow \infty)$.

Secondly, we prove that $* x$ is a fixed point of $\mathrm{T}$. Because of:

Then we have:

$$
\begin{aligned}
& \lim _{\mathrm{i} \rightarrow \infty} \mathrm{J}\left(\mathrm{x}_{*}-\mathrm{Tx}_{\mathrm{i}}, \mathrm{x}_{*}-\mathrm{Tx}_{*} \mid \mathrm{x}_{1}, \ldots, \mathrm{x}_{\mathrm{n}-1} \frac{\mathrm{t}}{\mathrm{k}(\alpha, \beta)}\right)=1, \\
& \lim _{\mathrm{i} \rightarrow \infty} \mathrm{J}^{\prime}\left(\mathrm{x}_{*}-\mathrm{Tx}_{\mathrm{i}}, \mathrm{x}_{*}-\mathrm{Tx}_{*} \mid \mathrm{x}_{1}, \ldots, \mathrm{x}_{\mathrm{n}-1}, \frac{\mathrm{t}}{\mathrm{k}(\alpha, \beta)}\right)=0 \forall \mathrm{t}>0 .
\end{aligned}
$$

Because $\mathrm{x}_{\mathrm{i}} \rightarrow \mathrm{x}_{*}($ when $\mathrm{i} \rightarrow) \Delta \mathrm{D}(\bullet, \bullet)$ and $\nabla(\bullet, \bullet)$ are equi-continuous at $(1,1)$ and $(0,0)$ respectively and:

$$
\begin{aligned}
& \mathrm{J}\left(\theta, \mathrm{x}_{*}-\mathrm{Tx}_{*} \mid \mathrm{x}_{1}, \ldots, \mathrm{x}_{\mathrm{n}-1}, \mathrm{t}\right)=1 \text { and } \\
& \mathrm{j}^{\prime}\left(\theta, \mathrm{x}_{*}-\mathrm{Tx}_{*}-\mathrm{Tx}_{*} \mid \mathrm{x}_{1}, \ldots, \mathrm{x}_{\mathrm{n}-1}, \mathrm{t}\right)=0 \text { we have } \\
& \lim _{\mathrm{i} \rightarrow\left(\mathrm{x}_{*}-T \mathrm{x}_{\mathrm{i}}, \mathrm{x}_{*}-\mathrm{Tx}_{*} \mid \mathrm{x}_{1}, \ldots, \mathrm{x}_{\mathrm{n}-1}, \frac{\mathrm{t}}{\mathrm{k}(\alpha, \beta)}\right)=1} \\
& \lim _{\mathrm{i} \rightarrow \infty} \mathrm{J}^{\prime}\left(\mathrm{x}_{*}-\mathrm{Tx}_{\mathrm{i}}, \mathrm{x}_{*}-\mathrm{Tx}_{*} \mid \mathrm{x}_{1}, \ldots, \mathrm{x}_{\mathrm{n}-1}, \frac{\mathrm{t}}{\mathrm{k}(\alpha, \beta)}\right)=0, \forall \mathrm{t}>0
\end{aligned}
$$

Hence:

$$
\mathrm{J}\left(\mathrm{x}_{*}-\mathrm{Tx}_{*}, \mathrm{x}_{*}-\mathrm{Tx}_{*} \mid \mathrm{x}_{1}, \ldots, \mathrm{x}_{\mathrm{n}-1}, \frac{\mathrm{t}}{\mathrm{k}(\alpha, \beta)}\right)
$$




$$
\begin{aligned}
& \geq \Delta\left(\begin{array}{l}
\mathrm{J}\left(\mathrm{x}_{*}-\mathrm{Tx}_{\mathrm{i}}, \mathrm{x}_{*}-\mathrm{Tx}_{*} \mid \mathrm{x}_{1}, \ldots, \mathrm{x}_{\mathrm{n}-1}, \frac{\mathrm{t}(1-\mathrm{k}(\alpha, \beta))}{\mathrm{k}(\alpha, \beta)}\right) \\
\mathrm{J}\left(\mathrm{Tx}_{\mathrm{i}}-\mathrm{Tx}_{*}, \mathrm{x}_{*}-\mathrm{Tx}_{*} \mid \mathrm{x}_{1}, \ldots, \mathrm{x}_{\mathrm{n}-1}, \mathrm{t}\right)
\end{array}\right) \\
& \geq \Delta\left(\begin{array}{l}
\mathrm{J}\left(\mathrm{x}_{*}-\mathrm{Tx}_{\mathrm{i}}, \mathrm{x}_{*}-\mathrm{Tx}_{*} \mid \mathrm{x}_{1}, \ldots, \mathrm{x}_{\mathrm{n}-1}, \frac{\mathrm{t}(1-\mathrm{k}(\alpha, \beta))}{\mathrm{k}(\alpha, \beta)}\right) \\
\mathrm{J}\left(\mathrm{x}_{\mathrm{i}}-\mathrm{x}_{*} \mathrm{x}_{*}-\mathrm{Tx}_{*} \mid \mathrm{x}_{1}, \ldots, \mathrm{x}_{\mathrm{n}-1}, \frac{\mathrm{t}}{\mathrm{k}(\alpha, \beta)}\right)
\end{array}\right), \\
& \mathrm{J}^{\prime}\left(\mathrm{x}_{*}-\mathrm{Tx}_{*}, \mathrm{x}_{*}-\mathrm{Tx}_{*} \mid \mathrm{x}_{1}, \ldots, \mathrm{x}_{\mathrm{n}-1}, \frac{\mathrm{t}}{\mathrm{k}(\alpha, \beta)}\right) \\
& \leq \nabla\left(\begin{array}{l}
\mathrm{J}^{\prime}\left(\mathrm{x}_{*}-\mathrm{Tx}_{\mathrm{i}}, \mathrm{x}_{*}-\mathrm{Tx}_{*} \mid \mathrm{x}_{1}, \ldots, \mathrm{x}_{\mathrm{n}-1}, \frac{\mathrm{t}(1-\mathrm{k}(\mathrm{a}, \beta))}{\mathrm{k}(\alpha, \beta)}\right) \\
\mathrm{J}^{\prime}\left(\mathrm{Tx}_{\mathrm{i}}-\mathrm{Tx}_{*}, \mathrm{x}_{*}-\mathrm{Tx}_{*} \mid \mathrm{x}_{1}, \ldots, \mathrm{x}_{\mathrm{n}-1}, \mathrm{t}\right)
\end{array}\right) \\
& \leq \nabla\left(\begin{array}{l}
\mathrm{J}^{\prime}\left(\mathrm{x}_{*}-\mathrm{Tx}_{\mathrm{i}}, \mathrm{x}_{*}-\mathrm{Tx}_{*} \mid \mathrm{x}_{1}, \ldots, \mathrm{x}_{\mathrm{n}-1}, \frac{\mathrm{t}(1-\mathrm{k}(\alpha, \beta))}{\mathrm{k}(\alpha, \beta)}\right) \\
\mathrm{J}^{\prime}\left(\mathrm{x}_{\mathrm{i}}-\mathrm{x}_{*}, \mathrm{x}_{*}-\mathrm{Tx}_{*} \mid \mathrm{x}_{1}, \ldots, \mathrm{x}_{\mathrm{n}-1}, \frac{\mathrm{t}}{\mathrm{k}(\alpha, \beta)}\right)
\end{array}\right) \\
& \operatorname{SoJ}\left(\mathrm{x}_{*}-\mathrm{Tx}_{*}, \mathrm{x}_{*}-\mathrm{Tx}_{*} \mid \mathrm{x}_{1}, \ldots, \mathrm{x}_{\mathrm{n}-1}, \frac{\mathrm{t}}{\mathrm{k}(\alpha, \beta)}\right) \rightarrow 1 \text {, } \\
& \mathrm{J}^{\prime}\left(\mathrm{x}_{*} \mathrm{Tx}_{*}, \mathrm{x}_{*}-\mathrm{Tx}_{*} \mid \mathrm{x}_{1}, \ldots, \mathrm{x}_{\mathrm{n}-1}, \frac{\mathrm{t}}{\mathrm{k}(\alpha, \beta)}\right) \rightarrow 0(\mathrm{i} \rightarrow \infty \forall \mathrm{t}>0) .
\end{aligned}
$$

By $((2),(3))$ and $((11,12))$ of Definition 11, we have $* * x=T x$ then:

If there exists a point $y \in X * \operatorname{such}$ that $* * y=T y$,

$$
\begin{aligned}
& J\left(x_{*}-y_{*}, x_{*}-y_{*} \mid x_{1}, \ldots, x_{n-1}, t\right)= \\
& J\left(T x_{*}-T x_{*}-T y_{*} \mid x_{1}, \ldots, x_{n-1}, t\right) \\
& \geq J\left(x_{*}-y_{*}, x_{*}-y_{*} \mid x_{1}, \ldots, x_{n-1}, \frac{t}{(\alpha, \beta)}\right) \\
& J^{\prime}\left(x_{*}-y_{*}, x_{*}-y_{*} \mid x_{1}, \ldots, x_{n-1}, t\right)=J^{\prime} \\
& \left(T x_{*}-T y_{*}, T x_{*}-T y_{*} \mid x_{1}, \ldots, x_{n-1}, t\right) \\
& \leq J^{\prime}\left(x_{*}-y_{*}, x_{*}-y_{*} \mid x_{1}, \ldots, x_{n-1}, \frac{t}{k^{2}(\alpha, \beta)}\right)
\end{aligned}
$$

In the same way, we obtain:

$$
\begin{aligned}
& \mathrm{J}\left(\mathrm{x}_{*}-\mathrm{y}_{*}, \mathrm{x}_{*}-\mathrm{y}_{*} \mid \mathrm{x}_{1}, \ldots, \mathrm{x}_{\mathrm{n}-1}, \mathrm{t}\right) \geq \mathrm{J} \\
& \left(\mathrm{x}_{*}-\mathrm{y}_{*}, \mathrm{x}_{*}-\mathrm{y}_{*} \mid \mathrm{x}_{1}, \ldots, \mathrm{x}_{\mathrm{n}-1}, \frac{\mathrm{t}}{\mathrm{k}^{2}(\alpha, \beta)}\right) \geq \ldots \geq
\end{aligned}
$$

$$
\begin{aligned}
& \mathrm{J}\left(\mathrm{x}_{*}-\mathrm{y}_{*}, \mathrm{x}_{*}-\mathrm{y}_{*} \mid \mathrm{x}_{1}, \ldots, \mathrm{x}_{\mathrm{n}-1}, \frac{\mathrm{t}}{\mathrm{k}^{2 \mathrm{n}}(\alpha, \beta)}\right), \\
& \mathrm{J}^{\prime}\left(\mathrm{x}_{*}-\mathrm{y}_{*}, \mathrm{x}_{*}-\mathrm{y}_{*} \mid \mathrm{x}_{1}, \ldots, \mathrm{x}_{\mathrm{n}-1}\right) \leq \mathrm{J}^{\prime} \\
& \left(\mathrm{x}_{*}-\mathrm{y}_{*}, \mathrm{x}_{*}-\mathrm{y}_{*} \mid \mathrm{x}_{1}, \ldots, \mathrm{x}_{\mathrm{n}-1} \frac{\mathrm{t}}{\mathrm{k}^{2}(\alpha, \beta)}\right), \\
& \leq \mathrm{J}^{\prime}\left(\mathrm{x}_{*}-\mathrm{y}_{*}, \mathrm{x}_{*}-\mathrm{y}_{*} \mid \mathrm{x}_{1}, \ldots, \mathrm{x}_{\mathrm{n}-1} \frac{\mathrm{t}}{\mathrm{k}^{2 \mathrm{n}}(\alpha, \beta)}\right) .
\end{aligned}
$$

Definition 11 , we have $\mathrm{x} *=\mathrm{y} *$. Therefore $\mathrm{x} *$ is the unique fixed point in $X$. Finally, we prove that the sequence $\left\{\mathrm{T}^{\mathrm{n}} \mathrm{x}_{0}\right\} \tau$ - converges to $x_{*}$ for any $\mathrm{x}_{0} \in \mathrm{X}$. Because of:

$$
\begin{aligned}
& \mathrm{J}\left(\mathrm{x}_{*}-\mathrm{T}^{\mathrm{n}} \mathrm{x}_{0}, \mathrm{x}_{*}-\mathrm{T}^{\mathrm{n}} \mathrm{x}_{0} \mid \mathrm{x}_{1}, \ldots, \mathrm{x}_{\mathrm{n}-1}, \mathrm{t}\right) \\
& =\mathrm{J}\left(\mathrm{Tx}_{*}-\mathrm{T}^{\mathrm{n}} \mathrm{x}_{0}, \mathrm{Tx}_{*}-\mathrm{T}^{\mathrm{n}} \mathrm{x}_{0} \mid \mathrm{x}_{1}, \ldots, \mathrm{x}_{\mathrm{n}-1}, \mathrm{t}\right) \\
& \geq \mathrm{J}\left(\mathrm{x}_{*}-\mathrm{T}^{\mathrm{n}-1} \mathrm{x}_{0}, \mathrm{x}_{*}-\mathrm{T}^{\mathrm{n}-1} \mathrm{x}_{0} \mid \mathrm{x}_{1}, \ldots, \mathrm{x}_{\mathrm{n}-1}, \frac{\mathrm{t}}{\mathrm{k}^{2}(\alpha, \beta)}\right) \\
& =J\left(\mathrm{Tx}_{*}-\mathrm{T}^{\mathrm{n}-1} \mathrm{x}_{0} \mathrm{Tx}_{*}-\left|\mathrm{T}^{\mathrm{n}-1} \mathrm{x}_{0}\right| \mathrm{x}_{1}, \ldots, \mathrm{x}_{\mathrm{n}-1}, \frac{\mathrm{t}}{\mathrm{k}^{2}(\alpha, \beta)}\right) \\
& \geq\left(\mathrm{x}_{*}-\mathrm{T}^{\mathrm{n}-2} \mathrm{x}_{0}, \mathrm{x}_{*}-\mathrm{T}^{\mathrm{n}-2} \mathrm{x}_{0} \mid \mathrm{x}_{1}, \ldots, \mathrm{x}_{\mathrm{n}-1}, \frac{\mathrm{t}}{\mathrm{k}^{4}(\alpha, \beta)}\right) \\
& \geq \ldots . \geq \mathrm{J}\left(\mathrm{x}_{*}-\mathrm{x}_{0}, \mathrm{x}_{*}-\mathrm{x}_{0} \mid \mathrm{x}_{1}, \ldots, \mathrm{x}_{\mathrm{n}-1}, \frac{\mathrm{t}}{\mathrm{k}^{2 \mathrm{n}}(\alpha, \beta)}\right) \\
& \mathrm{J}^{\prime}\left(\mathrm{x}_{*}-\mathrm{T}^{\mathrm{n}} \mathrm{x}_{0}, \mathrm{x}_{*}-\mathrm{T}^{\mathrm{n}} \mathrm{x}_{0} \mid \mathrm{x}_{1}, \ldots, \mathrm{x}_{\mathrm{n}-1}, \mathrm{t}\right) \\
& =\mathrm{J}^{\prime}\left(\mathrm{Tx}_{*}-\mathrm{T}^{\mathrm{n}} \mathrm{x}_{0}, \mathrm{Tx}_{*}-\mathrm{T}^{\mathrm{n}} \mathrm{x}_{0} \mid \mathrm{x}_{1}, \ldots, \mathrm{x}_{\mathrm{n}-1}, \mathrm{t}\right) \\
& \leq J^{\prime}\left(x_{*}-T^{n-1} x_{0}, x_{*}-T^{n-1} x_{0} \mid x_{1}, \ldots, x_{n-1}, \frac{t}{k^{2}(\alpha, \beta)}\right) \\
& =J^{\prime}\left(\mathrm{Tx}_{*}-\mathrm{T}^{\mathrm{n}-1} \mathrm{x}_{0}, \mathrm{Tx}_{*}-\mathrm{T}^{\mathrm{n}-1} \mathrm{x}_{0} \mid \mathrm{x}_{1}, \ldots, \mathrm{x}_{\mathrm{n}-1}, \frac{\mathrm{t}}{\mathrm{k}^{2}(\alpha, \beta)}\right) \\
& \leq J^{\prime}\left(x_{*}-T^{n-2} x_{0}, x_{*}-T^{n-2} x_{0} \mid x_{1}, \ldots, x_{n-1}, \frac{t}{k^{4}(\alpha, \beta)}\right) \\
& \leq \ldots \leq \mathrm{J}^{\prime}\left(\mathrm{x}_{*}-\mathrm{x}_{0}, \mathrm{x}_{*}-\mathrm{x}_{0} \mid \mathrm{x}_{1}, \ldots, \mathrm{x}_{\mathrm{n}-1}, \frac{\mathrm{t}}{\mathrm{k}^{2 \mathrm{n}}(\alpha, \beta)}\right)
\end{aligned}
$$

We have:

$$
\begin{aligned}
& \lim _{\mathrm{n} \rightarrow \infty} \mathrm{J}\left(\mathrm{x}_{*}-\mathrm{T}^{\mathrm{n}} \mathrm{x}_{0}, \mathrm{x}_{*}-\mathrm{T}^{\mathrm{n}} \mathrm{x}_{0} \mid \mathrm{x}_{1}, \ldots, \mathrm{x}_{\mathrm{n}-1}, \mathrm{t}\right) \\
& \geq \lim _{\mathrm{n} \rightarrow \infty} \mathrm{J}\left(\mathrm{x}_{*}-\mathrm{x}_{0}, \mathrm{x}_{*}-\mathrm{x}_{0} \mid \mathrm{x}_{1}, \ldots, \mathrm{x}_{\mathrm{n}-1}, \frac{\mathrm{t}}{\mathrm{k}^{2 \mathrm{n}}(\alpha, \beta)}\right)=1 \\
& \lim _{\mathrm{n} \rightarrow \infty} \mathrm{J}^{\prime}\left(\mathrm{x}_{*}-\mathrm{T}^{\mathrm{n}} \mathrm{x}_{0}, \mathrm{x}_{*}-\mathrm{T}^{\mathrm{n}} \mathrm{x}_{0} \mid \mathrm{x}_{1}, \ldots, \mathrm{x}_{\mathrm{n}-1}, \mathrm{t}\right) \\
& \leq \lim _{\mathrm{n} \rightarrow \infty} \mathrm{J}^{\prime}\left(\mathrm{x}_{*}-\mathrm{x}_{0}, \mathrm{x}_{*}-\mathrm{x}_{0} \mid \mathrm{x}_{1}, \ldots, \mathrm{x}_{\mathrm{n}-1}, \frac{\mathrm{t}}{\mathrm{k}^{2 \mathrm{n}}(\alpha, \beta)}\right)=0
\end{aligned}
$$


Applications: In what follows we shall utilize theorem 11 to study the existence and uniqueness of solution for linear Volterra integral equation on complete IF-n-IP space. We assume that $[a, b]$ is a fixed real interval. We define linear operation in $\mathrm{L}^{2}[\mathrm{a}, \mathrm{b}]$ :

$$
(\mathrm{x}+\mathrm{y})(\mathrm{t})=\mathrm{x}(\mathrm{t})+\mathrm{y}(\mathrm{t}),(\mathrm{ax})(\mathrm{t})=\mathrm{ax}(\mathrm{t})
$$

Then $\mathrm{L}^{2}[\mathrm{a}, \mathrm{b}]$ is a linear space. We lead n-inner product into $\mathrm{L}^{2}[\mathrm{a}, \mathrm{b}]$ :

$$
\begin{aligned}
& \left(x, y \mid x_{1}, \ldots, x_{n-1}\right)=\int_{a}^{b} x(t) \cdot y(t) \cdot x_{1}(t) \cdot x_{2}(t) \ldots x_{n-1}(t) d t \\
& \text { Hence }\left(x, y \mid x_{1}, \ldots, x_{n-1}\right) \text { is finite }
\end{aligned}
$$

Number, $(\bullet, \bullet \mid \bullet, \ldots, \bullet)$ satisfies all conditions of inner product and $\mathrm{L}^{2}[\mathrm{a}, \mathrm{b}]$ is an $\mathrm{n}$-inner product space by $(\bullet, \bullet \mid \bullet, \ldots, \bullet)$. Because $\mathrm{L}^{2}[\mathrm{a}, \mathrm{b}]$ is infinite dimensional and complete, then $\mathrm{L}^{2}[\mathrm{a}, \mathrm{b}]$ is a Hilbert space. Define a space $\left(\mathrm{L}^{2}[\mathrm{a}, \mathrm{b}], \mathrm{J}, \mathrm{J}, \Delta, \nabla\right)$, where:

$$
\begin{aligned}
& J, J^{\prime}: \widetilde{L^{2}}[\bar{a}, \bar{b}] \times \frac{\mathrm{L}^{2}[a, b] \times \ldots .1 \text { times }}{\mathrm{L}^{2}[a, b]} \rightarrow[0,1] \\
& J\left(x, y \mid x_{1}, \ldots, x_{n-1}, t\right)=\frac{t}{t+\left(x, y \mid x_{1}, \ldots, x_{n-1}\right)} \text { and } \\
& J^{\prime}\left(x, y \mid x_{1}, \ldots, x_{n-1}, t\right)=\frac{\left(x, y \mid x_{1}, \ldots, x_{n-1}\right)}{t+\left(x, y \mid x_{1}, \ldots, x_{n-1}\right)}
\end{aligned}
$$

Then $\left(\mathrm{L}^{2}[\mathrm{a}, \mathrm{b}], \mathrm{J}, \mathrm{J}, \Delta, \nabla\right)$ is a IFIP-space. In fact, let $\left\{\mathrm{x}_{\mathrm{n}}\right\}$ be a Cauchy sequence in $\left(\mathrm{L}^{2}[\mathrm{a}, \mathrm{b}], \mathrm{J}, \mathrm{J}, \Delta, \nabla\right)$. Then for any $\varepsilon>0, \lambda \in(0,1], \exists \mathrm{N}$, when $\mathrm{m}, \mathrm{n} \geq \mathrm{N}$, we have:

$$
\begin{aligned}
& J\left(x_{m}-x_{n}, x_{m}-x_{n} \mid x_{1}, \ldots, x_{n-1}, \varepsilon\right)> \\
& 1-\lambda, J^{\prime}\left(x_{m}-x_{n}, x_{m} \mid x_{1}, \ldots, x_{n-1}, \varepsilon\right) \leq \lambda
\end{aligned}
$$

Because of:

$$
\begin{aligned}
& \frac{J\left(x_{m}-x_{n}, x_{m}-x_{n} \mid x_{1}, \ldots, x_{n-1}, \varepsilon\right)=}{\varepsilon+\left(x_{m}-x_{n}, x_{m}-x_{n} \mid x_{1}, \ldots, x_{n-1}\right)} \\
& =\frac{\varepsilon}{\varepsilon+\int_{a}^{b}\left(x_{m}-x_{n}\right)(t) \cdot\left(x_{m}-x_{n}\right)(t) \cdot\left(x_{1}\right)(t) \cdot\left(x_{2}\right)(t) \ldots .\left(x_{n-1}\right)(t) d t} \\
& >1-\lambda
\end{aligned}
$$

And:

$$
\begin{aligned}
& J^{\prime}\left(x_{m}-x_{n}, x_{m}-x_{n} \mid x_{1}, \ldots, x_{n-1}, \varepsilon\right)= \\
& \frac{\left(x_{m}-x_{n}, x_{m}-x_{n} \mid x_{1}, \ldots, x_{n-1}\right)}{\varepsilon+\left(x_{m}-x_{n}, x_{m}-x_{n} \mid x_{1}, \ldots, x_{n-1}\right)} \\
& =\frac{\int_{a}^{b}\left(x_{1}\right)(t)\left(x_{2}\right)(t) \ldots . .\left(x_{n-1}\right)(t) d t}{\varepsilon+\int_{a}^{b}\left(x_{m}-x_{n}\right)(t)\left(x_{m}-x_{n}\right)(t) .} \leq \lambda
\end{aligned}
$$

We have:

$$
\int_{a}^{b}\left[\left(x_{m}-x_{n}\right)(t)\right]^{2} \cdot\left(x_{1}\right)(t) \cdot\left(x_{2}\right)(t) \ldots . .\left(x_{n-1}\right)(t) d t \rightarrow 0 .
$$

Then $x_{m}-x_{n} \rightarrow 0$ a.e. as $x_{1}, x_{2}, \ldots, x_{n-1}$ are not all zero. So $x_{n}$ is a Cauchy sequence in $\mathrm{L}^{2}[a, b]$. By the completeness of $\mathrm{L}^{2}[\mathrm{a}, \mathrm{b}]$, we have $\mathrm{x}_{\mathrm{n}} \rightarrow \mathrm{x}_{*} \in \mathrm{L}^{2}[\mathrm{a}, \mathrm{b}]$. Hence $\mathrm{X} * \in\left(\mathrm{L}^{2}[\mathrm{a}, \mathrm{b}], \mathrm{J}, \mathrm{J}, \Delta, \nabla\right) . \operatorname{So}\left(\mathrm{L}^{2}[\mathrm{a}, \mathrm{b}], \mathrm{J}, \mathrm{J}\right.$, $\Delta, \nabla)$ is a complete IF-n-IP space.

Theorem 15: Let $\left(\mathrm{L}^{2}[\mathrm{a}, \mathrm{b}], \mathrm{J}, \mathrm{J}, \Delta, \nabla\right)$ be a complete IF-n-IP space. Then the following conditions are satisfied:

$\int_{a}^{t}(x(s)-y(s)) d s \leq x(t)-y(t), \forall x(),. y(.) \in L^{2}[a, b]$

Let $\mathrm{T}$ be a linear mapping and defined as follows:

$(T x)(t)=f(t)+\lambda \int_{a}^{t} k(t, s) x(s) d s$

where $\mathrm{f} \in \mathrm{L}^{2}[\mathrm{a}, \mathrm{b}]$ is a given function, $\mathrm{k}(\mathrm{t}, \mathrm{s})$ is a continuous function defined on $\mathrm{a} \leq \mathrm{t} \leq \mathrm{b}, \mathrm{a} \leq \mathrm{s} \leq \mathrm{t}, \chi$ is a constant, we denote:

$$
\underset{\mathrm{a} \leq \mathrm{t} \leq \mathrm{b}, \mathrm{a} \leq \mathrm{s} \leq \mathrm{t}}{\mathrm{k}(\mathrm{t}, \mathrm{s})=\mathrm{M}}
$$

Then when $\chi \mathrm{M} \in(0,1), \mathrm{T}$ has a unique fixed point in $\mathrm{L}^{2}[\mathrm{a}, \mathrm{b}]$. Furthermore, for any $\mathrm{x}_{0} \in \mathrm{L}^{2}[\mathrm{a}, \mathrm{b}]$, the iterative sequence $\left\{\mathrm{T}^{\mathrm{n}} \mathrm{x}_{0}\right\} \tau$ - converges to the fixed point.

Proof:

$$
\begin{aligned}
& \left(\mathrm{Tx}-\mathrm{Ty}, \mathrm{v} \mid \mathrm{x}_{1}, \ldots, \mathrm{x}_{\mathrm{n}-1}\right)= \\
& \int_{a}^{b}(T x-T y)(t) \cdot(v)(t) \cdot\left(x_{1}\right)(t) \cdot\left(x_{2}\right)(t) \ldots .\left(x_{n-1}\right)(t) d t \\
& =\int_{a}^{b} \lambda\left(\int_{a}^{t}\left(k(t, s) x(s) d s-\int_{a}^{t} k(t, s) y(s) d s\right)\right) \\
& v(t) \cdot\left(x_{1}\right)(t) \cdot\left(x_{2}\right)(t) \ldots .\left(x_{n-1}\right)(t) d t \\
& =\lambda \int^{\mathrm{b}}\left(\int_{\mathrm{a}}^{\mathrm{t}} \mathrm{k}(\mathrm{t}, \mathrm{s})(\mathrm{x}(\mathrm{s})-\mathrm{y}(\mathrm{s})) \mathrm{ds}\right) \\
& { }^{a} v(t) \cdot\left(x_{1}\right)(t) \cdot\left(x_{2}\right)(t) \ldots .\left(x_{n-1}\right)(t) d t
\end{aligned}
$$

By the continuity of $\mathrm{k}(\mathrm{t}, \mathrm{s})$ and mean value theorem, $\exists \mathrm{t}_{1}, \mathrm{~s}_{1}{ }^{\prime}$ we have: 
$\left(T x-T y, v \mid x_{1}, \ldots ., x_{n-1}\right)=\lambda k\left(t_{1}, s_{1}\right) \int_{a}^{b}\left(\int_{a}^{t}(x(s)-y(s)) d s\right)$
$v(t) .\left(x_{1}\right)(t) .\left(x_{2}\right)(t) \ldots .\left(x_{n-1}\right)(t) d t$

By the condition (i), we have:

$$
\begin{aligned}
& \left(T x-T y, v \mid x_{1}, \ldots, x_{n-1}\right) \leq \lambda k \int_{a}^{b}\left(\int_{a}^{t}(x(s)-y(s)) d s\right) \\
& v(t) \cdot\left(x_{1}\right)(t) .\left(x_{2}\right)(t) \ldots\left(x_{n-1}\right)(t) d t \\
& =\lambda M\left(x-y, v \mid x_{1}, \ldots, x_{n-1}\right)
\end{aligned}
$$

Therefore:

$$
\begin{aligned}
& \left(\mathrm{Tx}-\mathrm{Ty}, \mathrm{v} \mid \mathrm{x}_{1}, \ldots, \mathrm{x}_{\mathrm{n}-1}\right) \leq \lambda M\left(\mathrm{x}-\mathrm{y}, \mathrm{v} \mid \mathrm{x}_{1}, \ldots, \mathrm{x}_{\mathrm{n}-1}\right) \\
& \frac{\mathrm{t}}{\mathrm{t}+\left(\mathrm{Tx}-\mathrm{Ty}, \mathrm{v} \mid \mathrm{x}_{1}, \ldots, \mathrm{x}_{\mathrm{n}-1}\right)} \geq \frac{\mathrm{t}}{\mathrm{t}+\lambda M\left(\mathrm{x}-\mathrm{y}, \mathrm{v} \mid \mathrm{x}_{1}, \ldots, \mathrm{x}_{\mathrm{n}-1}\right)}
\end{aligned}
$$

Because of $\chi \mathrm{M} \in(0,1)$, we get:

$$
\begin{aligned}
& \frac{t}{t+\left(T x-T y, v \mid x_{1}, \ldots ., x_{n-1}\right)} \geq \frac{\frac{t}{\lambda M}}{\frac{t}{\lambda M}+\left(x-|y, v| x_{1}, \ldots, x_{n-1}\right)} \\
& \mathrm{J}\left(\mathrm{Tx}-\mathrm{Ty}, \mathrm{v} \mid \mathrm{x}_{1}, \ldots, \mathrm{X}_{\mathrm{n}-1}\right) \geq \mathrm{J}\left(\mathrm{x}-\mathrm{y}, \mathrm{v} \mid \mathrm{x}_{1}, \ldots, \mathrm{x}_{\mathrm{n}-1}, \frac{\mathrm{t}}{\lambda \mathrm{M}}\right) \text { and } \\
& \left(T x-T y, v \mid x_{1}, \ldots, x_{n-1}\right) \leq \lambda M\left(x-y, v \mid x_{1}, \ldots, x_{n-1}\right) \\
& \frac{\mathrm{t}}{\left(\mathrm{Tx}-\mathrm{Ty}, \mathrm{v} \mid \mathrm{x}_{1}, \ldots, \mathrm{x}_{\mathrm{n}-1}\right)} \geq \frac{\mathrm{t}}{\lambda \mathrm{M}\left(\mathrm{x}-\mathrm{y} \mid \mathrm{x}_{1}, \ldots, \mathrm{x}_{\mathrm{n}-1}\right)} \\
& 1+\frac{\mathrm{t}}{\left(\mathrm{Tx}-\mathrm{Ty}, \mathrm{v} \mid \mathrm{x}_{1}, \ldots, \mathrm{x}_{\mathrm{n}-1}\right)} \geq 1+\frac{\mathrm{t}}{\lambda \mathrm{M}\left(\mathrm{x}-\mathrm{y} \mid \mathrm{x}_{1}, \ldots, \mathrm{x}_{\mathrm{n}-1}\right)} \\
& \frac{\left(\mathrm{Tx}-\mathrm{Ty}, \mathrm{v} \mid \mathrm{x}_{1}, \ldots, \mathrm{x}_{\mathrm{n}-1}\right)+\mathrm{t}}{\left(\mathrm{Tx}-\mathrm{Ty}, \mathrm{v} \mid \mathrm{x}_{1}, . ., \mathrm{x}_{\mathrm{n}-1}\right)} \geq \frac{\lambda \mathrm{M}\left(\mathrm{x}-\mathrm{y}, \mathrm{v} \mid \mathrm{x}_{1}, \ldots, \mathrm{x}_{\mathrm{n}-1}\right)+\mathrm{t}}{\lambda \mathrm{M}\left(\mathrm{x}-\mathrm{y}, \mathrm{v} \mid \mathrm{x}_{1}, \ldots, \mathrm{x}_{\mathrm{n}-1}\right)} \\
& \frac{\left(T x-T y, v \mid x_{1}, \ldots, x_{n-1}\right)}{t+\left(T x-T y, v \mid x_{1}, \ldots, x_{n-1}\right)} \leq \frac{\lambda M\left(x-y, v \mid x_{1}, \ldots, x_{n-1}\right)}{t+\lambda M\left(x-y, v \mid x_{1}, \ldots, x_{n-1}\right)} \\
& \frac{\left(T x-T y, v \mid x_{1}, \ldots, x_{n-1}\right)}{t+\left(T x-T y, v \mid x_{1}, \ldots, x_{n-1}\right)} \leq \frac{\lambda M\left(x-y, v \mid x_{1}, \ldots, x_{n-1}\right)}{t+\lambda M\left(x-y, v \mid x_{1}, \ldots, x_{n-1}\right)} \\
& =\frac{\left(x-y, v \mid x_{1}, \ldots ., x_{n-1}\right)}{\frac{t}{\lambda M}+\left(x-y, v \mid x_{1}, \ldots, x_{n-1}\right)} \\
& J^{\prime}\left(T_{x}-T y, v \mid x_{1}, \ldots, x_{n-1}, t\right) J^{\prime}\left(x-y, v \mid x_{1}, \ldots, x_{n-1}, \frac{t}{\lambda M}\right)
\end{aligned}
$$

By Theorem 11, $\mathrm{T}$ has a unique fixed point in $\mathrm{L}^{2}$ $[a, b]$. Furthermore, for any $\mathrm{x}_{0} \in \mathrm{L}^{2}[\mathrm{a}, \mathrm{b}]$, the iterative sequence $\left\{\mathrm{T}^{\mathrm{m}} \mathrm{x}_{0}\right\} \tau-$ converges to the fixed point. This completes the proof.

\section{REFERENCES}

Atanassov, K.T., 1986. Intuitionistic fuzzy sets. Fuzzy Sets Syst., 20: 87-96. DOI: 10.1016/S01650114(86)80034-3

Bag, T. and S.K. Samanta, 2003. Finite dimensional fuzzy normed linear spaces. J. Fuzzy Math., 11: 687-705.

Cheng, S.C. and J.N. Mordeson, 1994. Fuzzy linear operators and fuzzy normed linear spaces. Bull. Calcutta Math. Soc., 86: 429-436.

Cho, Y.J., 2001. Theory of 2-Inner Product Spaces. 1st Edn., Nova Science Publishers, Inc., Huntington, New York, ISBN-10: 1560729007, pp: 330.

Cho, Y.J., M. Matic and J. Pecaric, 2002. Inequalities of Hlawka's type in n-inner product spaces. Commun. Korean Math. Soc., 17: 583-592. Diminnie, C.R., S. Gahler and A. White, 1977. 2inner product spaces. II, Demonstratio Math., 10: 169-188.

El-Abyad, A.M. and H.M. El-Hamauly, 1991. Fuzzy inner product spaces. Fuzzy Sets Syst., 44: 309326. Felbin, C., 1992. Finite dimensional fuzzy normed linear space. Fuzzy Sets Syst., 48: 239248. DOI: 10.1016/0165-0114(92)90338-5

Felbin, C., 1993. The completion of fuzzy normed linear space. J. Math. Anal. Appl., 174: 428-440. DOI: $10.1006 /$ jmaa.1993.1128

Felbin, C., 1999. Finite dimensional fuzzy normed linear space II. J. Anal., 7: 117-131.

Gahler, S., 1964. Lineare 2-normierte Raume. Math. Nachr., 28: 1-43. DOI: 10.1002/mana.19640280102

Gunawan, H. and M. Mashadi, 1986. On n-normed spaces. Int. J. Math. Math. Sci., 27: 631-639. DOI: 10.1155/S0161171201010675

Katsaras, A.K., 1984. Fuzzy topological vector spaces. Fuzzy Sets Syst., 12: 143-154. DOI: 10.1016/01650114(84)90034-4

Krishna, S.V. and K.K.M. Sarma, 1994. Separation of fuzzy normed linear spaces. Fuzzy Sets Syst., 63: 207-217. DOI: 10.1016/0165-0114(94)90351-4

Malceski, R., 1997. Strong n-convex n-normed spaces. Mat. Bilten., 21: 81-102.

Misiak, A., 1989a. N-inner product spaces. Math. Nachr., $\quad$ 140: 299-319. DOI: 10.1002/mana.19891400121 
Misiak, A., 1989b. Orthogonality and orthonormality in n-inner product spaces. Math. Nachr., 143: 249261. DOI: $10.1002 /$ mana.19891430119

Narayanan, A.L. and S. Vijayabalaji, 2005. Fuzzy nnormed linear space. Int. J. Math. Math. Sci., 24: 3963-3977. DOI: 10.1155/IJMMS.2005.3963

Rhie, G.S., B.M. Choi and D.S. Kim, 1997. On the completeness of fuzzy normed linear spaces. Math Japon., 45: 33-37.
Vijayabalaji, S. and N. Thillaigovindan, 2007. Fuzzy ninner product space. Bull. Korean Math. Soc., 43: 447-459.

Vijayabalaji, S., N. Thillaigovindan and Y.B. Jun, 2007. Intuitionistic fuzzy nnormed linear space. Bull. Korean Math. Soc., 44: 291-308.

Zadeh, L.A., 1965. Fuzzy Sets. Infor. Control., 89: 338- 